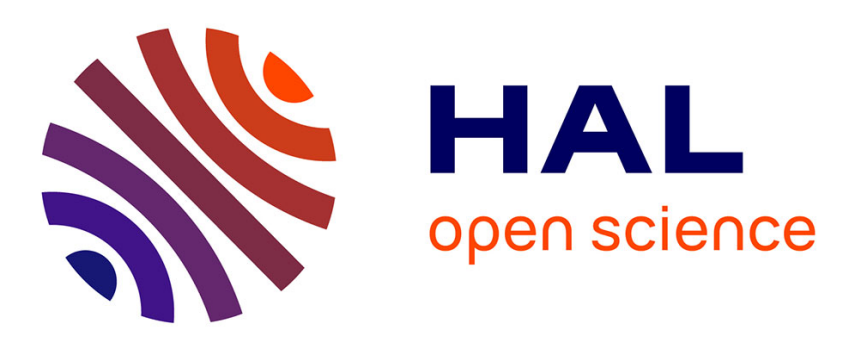

\title{
Impact of the volume of rooms on shock wave propagation within a multi-chamber system
}

Baptiste Julien, Isabelle Sochet, Thierry Vaillant

\section{To cite this version:}

Baptiste Julien, Isabelle Sochet, Thierry Vaillant. Impact of the volume of rooms on shock wave propagation within a multi-chamber system. Shock Waves, 2016, 26 (2), 10.1007/s00193-015-0603-2 . hal-01389899

\section{HAL Id: hal-01389899 \\ https://hal.science/hal-01389899}

Submitted on 30 Oct 2016

HAL is a multi-disciplinary open access archive for the deposit and dissemination of scientific research documents, whether they are published or not. The documents may come from teaching and research institutions in France or abroad, or from public or private research centers.
L'archive ouverte pluridisciplinaire HAL, est destinée au dépôt et à la diffusion de documents scientifiques de niveau recherche, publiés ou non, émanant des établissements d'enseignement et de recherche français ou étrangers, des laboratoires publics ou privés. 


\title{
Impact of the Volume of the Rooms on the Shock Wave Propagation within a Multi-Chamber System
}

\author{
Baptiste Julien • Isabelle Sochet - Thierry Vaillant
}

Received: date / Accepted: date

\begin{abstract}
The behavior of a shock wave generated by a hemispherical gaseous charge and propagating within a confined multi-chamber system is analyzed through the evolution of some of the shock parameters (maximum overpressure and positive impulse). Moreover, the impact of a variation in the volume of the rooms on the pressure history inside the building is also studied.

Several small-scale experiments have been carried out using an adjustable model representative of a pyrotechnic workshop.

The experimental results show that the pressure histories are very complex. Yet, using a global approach, we were able to link the evolution of the arrival time of the shock wave within the building with the reference obtained in free field. New parameters were developed to best fit the experimental maximal overpressure in the cells and in the corridor leading to two predictive laws used to estimate the maximal overpressure in the model.
\end{abstract}

Keywords Confined room - Shock wave - Detonation · Experiment $\cdot$ Multi-chamber

B. Julien · T. Vaillant

Direction Générale de l'Armement, Techniques Terrestres Rocade Est, Echangeur de Guerry - 18021 Bourges Cedex France

I. Sochet

INSA Centre Val de Loire - Université d'Orléans - PRISME

EA 4229 - 88 boulevard Lahitolle, 18000 Bourges - France

Tel.: +33-2-48484046

E-mail: isabelle.sochet@insa-cvl.fr

\section{Introduction}

Security is a real and major concern, especially when explosives are involved. These explosives threats can be of accidental nature or due to malevolent actions. To address this security issue, an accurate prediction of the behavior of shock waves generated by a detonation propagating inside a building is required.

Extensive knowledge regarding shock waves in free field can be found in the literature so that their behavior is now well known [1-3]. Analytical, empirical or numerical tools have been developed to predict the pressure flow generated by the detonation of an explosive in free field. However, there is very little data available in the open literature for confined configuration and the tools developed in free field do not apply in this case or are limited. Much more information can be found on semiconfined configurations such as tunnels [4] or urban terrain [5] that can provide us with a hint regarding the behavior of shock waves in confined environment. Yet, these works cannot be compared to the experiments presented in this article. The use of numerical simulations is also limited in complex confined environment for several reasons. An accurate prediction of the physical phenomena under investigation requires a very fine mesh, especially due to the reflections encountered in confined configuration. Moreover, its complexity often requires a full $3 \mathrm{D}$ simulation thus the running time of a simulation can be prohibitive.

The few authors who evaluate the behavior of shock waves in a confined environment often use visualization techniques such as Schlieren photography [6].

This study focuses on the behavior of a shock wave within a confined multi-chamber system and on the impact of the volume of the rooms on the pressure history inside the building. Several small-scale experiments are 
carried out using an adjustable model of a four-roomed single-story building, representative of a pyrotechnic workshop. Small scale experiments have multiple advantages like low cost, precise laboratory experiments, controlled test conditions and are particularly suitable for parametric studies. Moreover, as the dimensions are reduced, the mass of explosive required for experiments is also reduced providing more secure and less complex experiments $[7,8]$.

The two configurations presented in this article are designed to evaluate the effect of the volume of the rooms on the shock wave propagation. Five different lengths are tested in the first configuration and three widths in the second. The shock wave propagation is analyzed through the evolution of some of the shock parameters (arrival time, maximum overpressure and positive impulse).

\section{Experimental setup}

As was previously mentioned, the experimental set-up is based on an adjustable model of a four-roomed singlestory building, representative of a pyrotechnic workshop. The rooms are all aligned on the same side of the model and connected to a single corridor. The detonation is generated by a hemispherical charge of a propane-oxygen stoichiometric mixture located at the center of cell 2. Three charges are fired in the tests, a $30 \mathrm{~mm}$ radius charge, a $47 \mathrm{~mm}$ radius charge and a $67 \mathrm{~mm}$ radius charge. Pressure histories are recorded over a $150 \mathrm{~ms}$ period with fifteen pressure transducers distributed on the ground in the four rooms and in the corridor. A more detailed description of the experimental setup can be found in a previous article [9]. The model, made of Medium density fiberboard, was designed with the help of DGA Land Systems (Figure 1). The generic layout of the pressure transducers within the model is given in figure 2. As can be seen in figure 2 , the majority of the pressure transducers are shielded from the charge by the structure of the model. When a point is shielded from the charge by an obstacle in a fully confined environment, the shock wave cannot hit it directly. The shortest path leading from the charge to this point can be predicted by studying the reflections and diffractions the shock wave has to endure to reach this point. Using this path, a new set of scaled distances $\left(Z_{\text {conf }}\right)$ adapted to confined structure has been calculated. The methodology is more precisely described in a previous article [9]. In our two configurations the shortest path is always obtained with a diffraction.

Reference curves were established in free field and will be used to analyze pressure histories in a confined environment. The maximal overpressure $(\Delta P i)$, the arrival

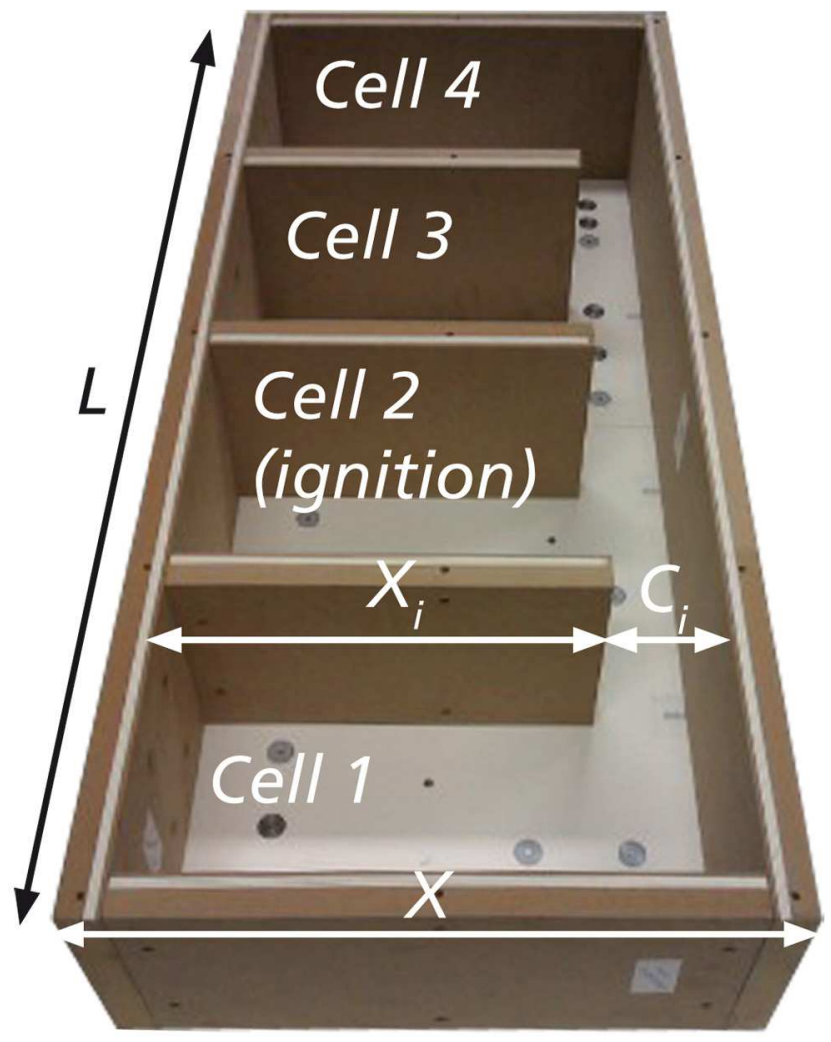

Fig. 1 Photograph of the model in the first configuration, $\mathrm{X}$ : width of the model, L: length of the model, $\mathrm{Ci}$ : interior corridor, Xi: length of the cells

\begin{tabular}{|c|c|c|c|}
\hline $\begin{array}{c}\otimes \mathrm{G} 1 \\
\mathrm{G} 2 \otimes \\
\\
\text { C } 1\end{array}$ & $\mathrm{C}_{2}^{\mathrm{G} 6 \otimes}$ & $\begin{array}{c}\otimes \mathrm{G} 910 \otimes \\
\text { C } 3\end{array}$ & $\begin{array}{c}\otimes_{\mathrm{G} 13} \mathrm{G}_{14} \otimes \\
\text { C } 4\end{array}$ \\
\hline $\mathrm{G} 3 \otimes$ & $\mathrm{G} 7 \otimes$ & $\mathrm{G} 11 \otimes$ & $\mathrm{G} 15^{\otimes}$ \\
\hline $\mathrm{G} 4 \otimes$ & $\mathrm{G} 8 \otimes$ & $\mathrm{G} 12 \otimes$ & $\mathrm{G} 16 \otimes$ \\
\hline
\end{tabular}

Fig. 2 Generic layout of the pressure transducers within the model

time $(t a)$ and the positive impulse $\left(I^{+}\right)$are taken down from the pressure histories. All these data are then processed and a polynomial is calculated for each of the three previous characteristics as a function of the scaled distance $(Z)[9]$. The scaled distance $Z\left(m . \mathrm{kg}^{-1 / 3}\right)$ is defined by $Z=R \cdot M^{-1 / 3}$ where $R$ is the radial distance from the charge center and $M$ the mass of the gas. However, to easily compare these polynomials with other studies, the mass of the gas is calculated for a spherical volume instead of the hemispherical volume used in the experiments. Thus, the studied field ranges from $0.969 \mathrm{~m} . \mathrm{kg}^{-1 / 3}$ to $14.116 \mathrm{~m} . \mathrm{kg}^{-1 / 3}$ : 
- Overpressure $(\Delta P i$, bar $)$ :

$$
\begin{array}{r}
\ln \left(\Delta P_{i}\right)=1.4745-1.412 \ln (Z)-0.3695 \ln (Z)^{2} \\
+0.2182 \ln (Z)^{3}-0.034 \ln (Z)^{4}
\end{array}
$$

- Scaled arrival time $\left(\overline{t_{a}}=t_{a} \cdot M^{-1 / 3}, \mathrm{~ms}_{\mathrm{kg}} \mathrm{k}^{-1 / 3}\right)$ :

$$
\begin{array}{r}
\ln \left(\overline{t_{a}}\right)=-0.3216+1.8031 \ln (Z)-0.0887 \ln (Z)^{2} \\
-0.0368 \ln (Z)^{3}+0.0081 \ln (Z)^{4}
\end{array}
$$

- Scaled positive impulse $\left(\overline{I^{+}}=I^{+} \cdot M^{-1 / 3}\right.$, $10^{2}$. bar.ms. $\left.\mathrm{kg}^{-1 / 3}\right)$ :

$$
\begin{aligned}
\ln \left(\overline{I^{+}}\right)=4.861 & -0.9979 \ln (Z)+0.3021 \ln (Z)^{2} \\
& -0.1935 \ln (Z)^{3}+0.0322 \ln (Z)^{4}
\end{aligned}
$$

The polynomials calculated in this study are quite the same than the ones from Sauvan [8] and Trélat [10] for similar experiences with a propane-oxygen stoichiometric mixture.

\subsection{Configuration 1}

The purpose of this configuration is to evaluate the effect of the length $\mathrm{X}$ of the cells on the shock waves propagation. Five lengths $\mathrm{X}$ have been tested. The generic sketch of the first configuration and the corresponding dimensions are presented in figure 3 and table 1 . The parameter $\mathrm{h}$ in table 1 gives the height of the model and is therefore not shown in figure 3 .

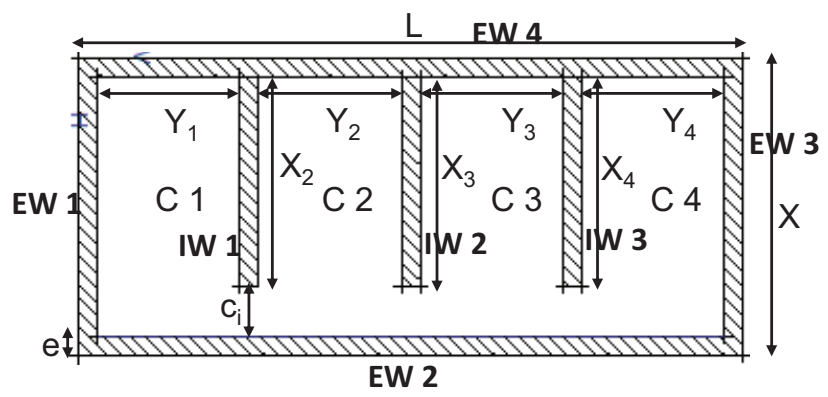

Fig. 3 Generic sketch of configuration 1

\subsection{Configuration 2}

The second configuration aims at evaluating the effect of the width $\mathrm{Y}$ of the cells on the shock waves propagation. Two different widths $\mathrm{Y}$ have been tested. The generic sketch of configuration 2 and the corresponding dimensions are presented in figure 4 and table 2 . In table $2, \mathrm{~h}$ is the height of the model. It can be noted that
Table 1 Configuration 1 dimensions

\begin{tabular}{|c|c|c|c|c|c|}
\hline \multicolumn{5}{|c|}{ Configuration 1 } \\
\hline \multirow{2}{*}{} & \multicolumn{5}{|c|}{1310} \\
\cline { 2 - 6 } & 1.1 & 1.2 & 1.3 & 1.4 & 1.5 \\
\hline $\mathrm{L}$ & \multicolumn{5}{|c|}{386} \\
\hline $\mathrm{X}$ & \multicolumn{5}{|c|}{240} \\
\hline$X_{2,3,4}$ & 328 & 369 & 410 & 431 & 451 \\
\hline$c_{i}$ & 182 & 141 & 100 & 79 & 59 \\
\hline $\mathrm{e}$ & \multicolumn{5}{|c}{} \\
\hline $\mathrm{h}$ & \multicolumn{5}{|c|}{280} \\
\hline $\mathrm{Yi}$ & \multicolumn{5}{|c|}{} \\
\hline
\end{tabular}

for this configuration, the length of the cells is the same as in configuration 1.3. Therefore, configuration 1.3 provides us with another width to be compared with the two evaluated in configuration 2 .

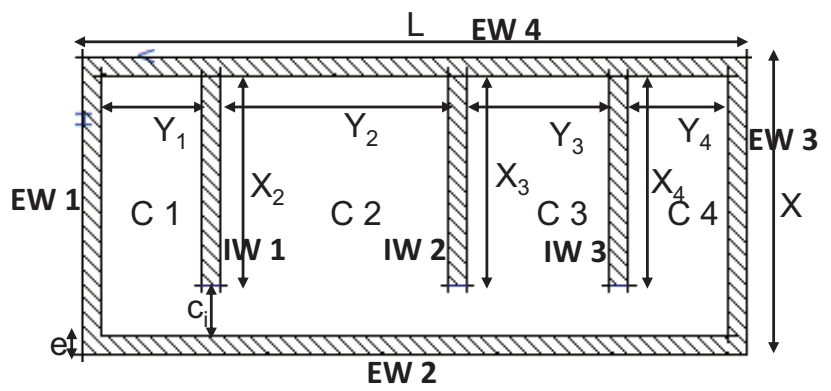

Fig. 4 Generic sketch of configuration 2

Table 2 Configuration 2 dimensions

\begin{tabular}{|c|c|c|}
\hline \multicolumn{3}{|c|}{ Configuration 2 } \\
\hline \multirow{2}{*}{} & \multicolumn{2}{|c|}{ distance $(\mathrm{mm})$} \\
\cline { 2 - 3 } & 2.1 & 2.2 \\
\hline $\mathrm{L}$ & \multicolumn{2}{|c|}{1310} \\
\hline $\mathrm{X}$ & \multicolumn{2}{|c|}{586} \\
\hline$Y_{2}$ & 448 & 616 \\
\hline$Y_{1,4}$ & 196 & 112 \\
\hline$Y_{3}$ & \multicolumn{2}{|c|}{280} \\
\hline $\mathrm{e}$ & \multicolumn{2}{|c|}{38} \\
\hline $\mathrm{h}$ & \multicolumn{2}{|c|}{240} \\
\hline$X_{2,3,4}$ & \multicolumn{2}{|c|}{410} \\
\hline$c_{i}$ & \multicolumn{2}{|c|}{100} \\
\hline
\end{tabular}

\section{Impact of the volume on the shock wave parameters within the detonation cell}

The case of the cell where the detonation is initiated is different from that of the other cells since the gauges 
are all in direct view of the charge. Thus we chose to analyze first the impact of the volume within this cell. In this article, it was chosen to only present the pressure histories recorded with a $67 \mathrm{~mm}$ radius charge. Yet, the results related to the three masses tested during the experiments will be discussed.

\subsection{Gauges G6 and G7}

Gauges G6 and G7 are both located inside the detonation cell at almost the same distance from the center of the charge but at different locations. Gauge G6 is indeed located in the corner at the end of the cell while Gauge G7 is at the middle of the exit of the cell (Figure 2).

The pressure histories recorded at gauge G6 in free field and in configuration 1.3 are plotted as a function of the scaled distance in figure 5 and figure 6 .

The overpressure recorded in free field slowly decreased

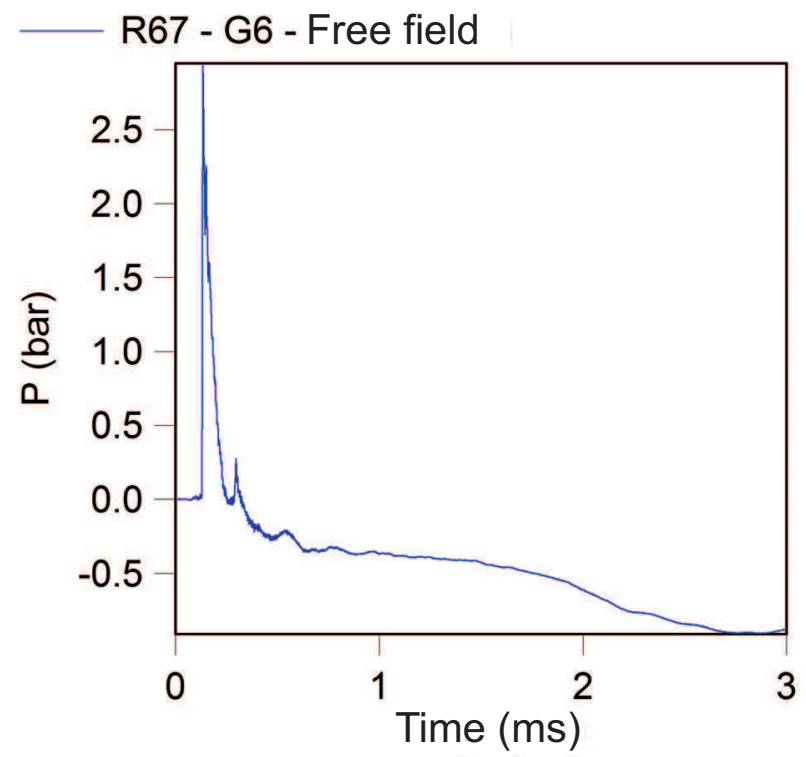

Fig. 5 Pressure history recorded at gauge G6 in free field for a $67 \mathrm{~mm}$ radius gaseous charge

after $1 \mathrm{~ms}$ (Figure 5). This could be due to the thermal drift associated with the sensor exposure to the radiation generated by the detonation. This phenomenon, also encountered by others authors like Mainiero [11], can alter the pressure history, especially in a closed configuration where the hot gazes are trapped by the structure.

In configuration 1.3, the pressure history is much more complex and presents several reflected peaks (Figure

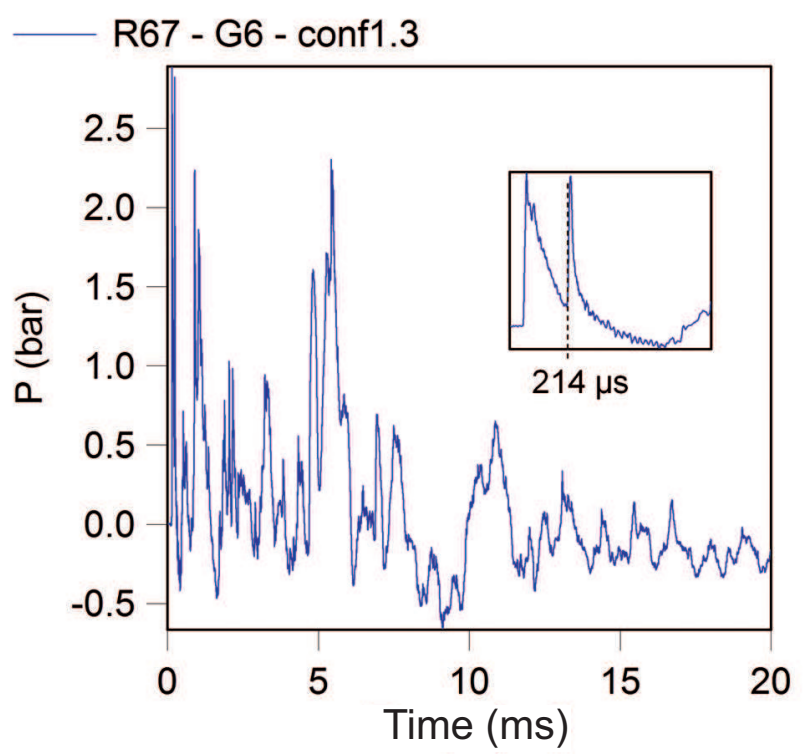

Fig. 6 Pressure history recorded at gauge G6 in configuration 1.3 for a $67 \mathrm{~mm}$ radius gaseous charge

$6)$. The maximal overpressure is obtained for the incident peak, then the amplitude of the various reflected peaks is lower. This was also observed by Sinha [12] for a sensor placed in the detonation room and with no indications regarding the origins of these reflected peaks. As this gauge is inside the detonation cell, the incident peak pressure is exactly the same as the one observed in free field with an arrival time of $124 \mu \mathrm{s}$. However, the expansion of the incident shock wave is aborted following arrival of the first reflecting peak at $214 \mu \mathrm{s}$.

An analytic study of the reflections observed at this gauge was then carried out to determine the nature of this peak. First of all, the Mach stems calculation showed that no direct Mach stem could hit the gauge G6. For example, wall IW2 is at $137 \mathrm{~mm}$ from the center of the explosive. The direct distance from the charge to the wall HOB is therefore $137 \mathrm{~mm}$. Setting the origin of the set of axes at the center of the charge, the coordinates of gauge $\mathrm{G} 6$ are $(102.5 \mathrm{~mm},-107 \mathrm{~mm})$, $r_{i}$, the distance from the charge to the point of interest, is thus $120.5 \mathrm{~mm}$. The minimal angle of incidence which enables Mach reflection is $39^{\circ}$. Moreover, since the Mach stem shall reach gauge G6, the angle of incidence is at maximum $\beta=\arctan \left(\frac{r_{i}}{H O B}=36.8^{\circ}\right)$ (Figure 7). Therefore, no Mach reflection can occur on wall IW2 before the wave reach gauge G6. Using the same approach for walls IW1 and EW4, it appears that the angles of incidence are at maximum $35.6^{\circ}$ and $27.8^{\circ}$.

Moreover, the position of gauge G6 does not allow a normal reflection, thus the first reflected peak comes from an oblique reflection. The comparison with a roof- 


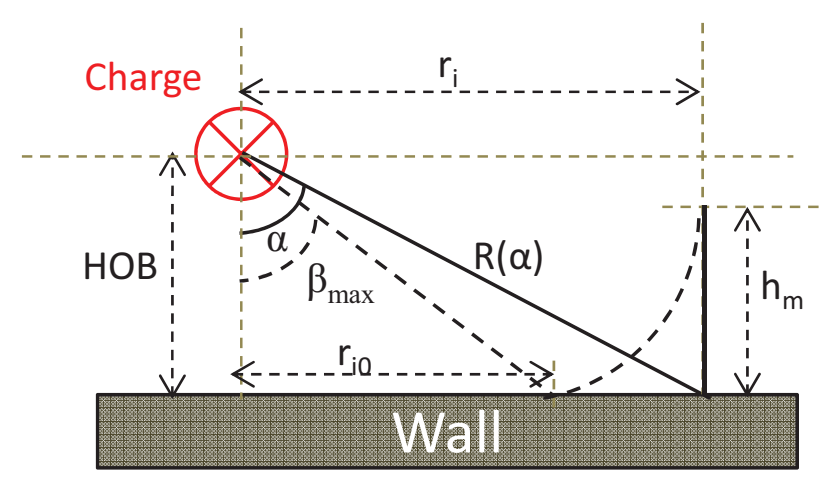

Fig. 7 Mach reflection

less test (Figure 8) indicates that this reflected peak is not due to the roof and the analysis of the oblique reflections on the ground shows that the reflection comes from the right hand wall in cell 2 , with cell 2 displayed as in figure 2. Indeed, the estimated arrival time of this reflected shock wave at gauge G6 is $258 \mu$ s which is consistent with the value noted in the roofless test. The approach leading to the estimation of the arrival time, as it is based on the free field parameters, ignores the effect of the expansion of the detonation products in a confined environment on the shock wave propagation. Indeed, in free field, the expansion of the detonation products is hemispherical with a ratio to the initial radius of 2.7 [13]. Yet, in the model, the expansion of the detonation products is limited by the geometry of the structure. And since the detonation products and air have different properties, the shock wave does not travel at the same celerity within both media. This may be one of the reasons explaining the discrepancies observed between the arrival time estimated by calculations and the one obtained in the roofless test with the value observed in configuration 1.3.

In semi-confined environment, new rarefaction waves are generated by the edges at the top of the walls. These rarefaction waves eventually catch up with the reflected shock and limit its amplitude [14]. This may explain the discrepancies between the reflected peaks with or without the roof.

The pressure histories recorded at gauge G7 have been plotted in free field and in configuration 1.3 (Figures 9 and 10).

Gauge G7, as gauge G6, is located inside the detonation cell. The incident peak pressure, that has an arrival time of $131 \mu \mathrm{s}$, is thus exactly the same as the one observed in free field. The first reflected peak appears at $285 \mu \mathrm{s}$.

The pressure history recorded in free field shows a weak reflected peak during the expansion phase. It is proba-

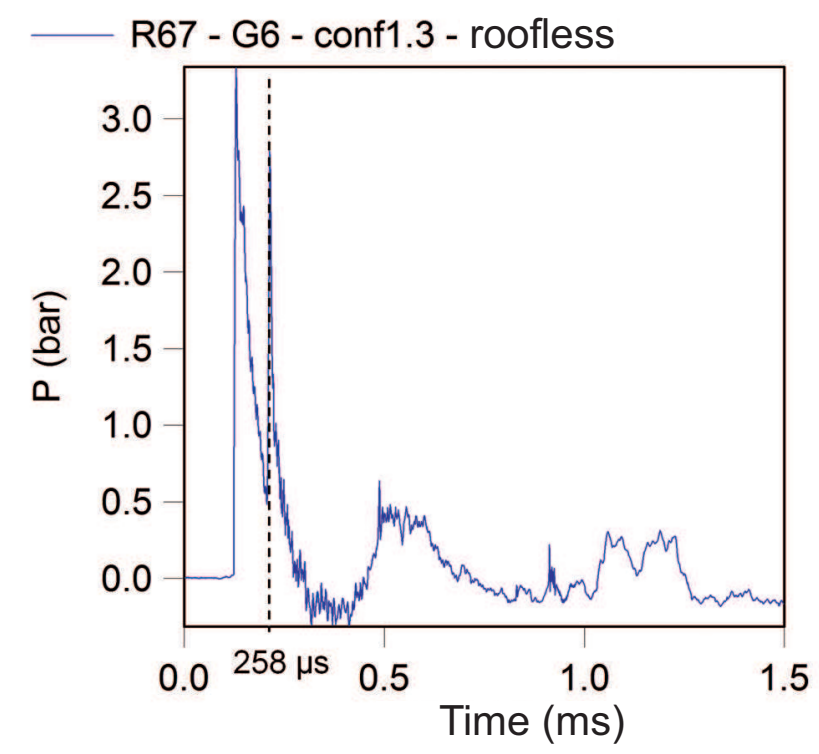

Fig. 8 Pressure history recorded at gauge G6 in configuration 1.3 roofless for a $67 \mathrm{~mm}$ radius gaseous charge

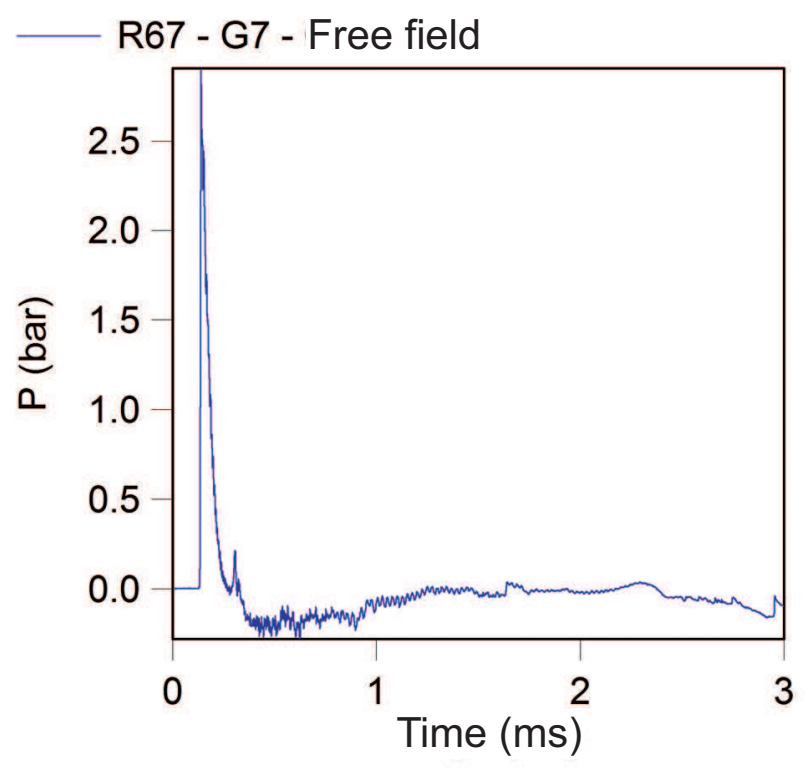

Fig. 9 Pressure history recorded at gauge G7 in free field for a $67 \mathrm{~mm}$ radius gaseous charge

bly caused by the implosion of the gaseous charge. Indeed, during the detonation of a hemispherical gaseous charge, initiated at its center, the shock wave propagates spherically through the gaseous medium. When the shock wave reaches the interface between gas and air, expansion waves travelling backward are generated [14]. Eventually, these waves interact at the center of the hemisphere and form a shock wave propagating in the same direction as the initial detonation wave.

The same analytic analysis that the one used previously for gauge G6 has been done with gauge G7. With 


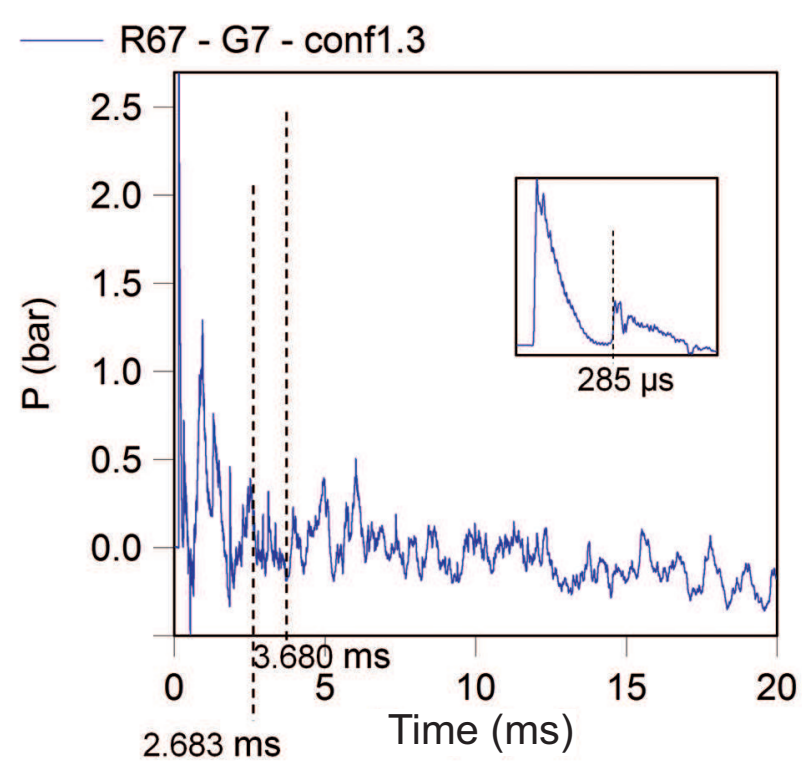

Fig. 10 Pressure history recorded at gauge G7 in configuration 1.3 for a $67 \mathrm{~mm}$ radius gaseous charge

the model orientated as presented in figure 2, the Mach stem calculations showed that Mach stems could develop along the left and right walls of cell 2. However, the height of this Mach stems are respectively of $0.9 \mathrm{~mm}$ and $1.2 \mathrm{~mm}$, which are not enough to impact gauge G7. Two normal reflections on walls EW2 and EW4 may reach gauge G7. EW2 is at a stand-off distance of $307.5 \mathrm{~mm}$. For a $67 \mathrm{~mm}$ radius charge, this translates into a scaled distance $Z(R)=2.551 \mathrm{~m} . \mathrm{kg}^{-1 / 3}$. Using this value in equation (2), the arrival time of the incident shock wave at wall EW2 is $\overline{t_{a}}=3.543 \mathrm{~ms} \mathrm{~kg}^{-1 / 3}$. Equations (4) and (5) can then be used to calculate the Mach number $M_{0}$ of the incident shock wave at the wall $M_{0}=2.117$. The Mach number of the reflected shock wave can now be deduced from equation (6) $M_{r}=1.790$.

$M_{0}=\frac{D_{0}}{a_{0}}$

$D_{0}=\frac{Z}{\overline{t_{a}} \cdot 10^{-3}}$

$M_{r}=\sqrt{\frac{2 \gamma M_{0}^{2}-(\gamma-1)}{(\gamma-1) M_{0}^{2}+2}}$

Using this reflected Mach number as an incident Mach number, the scaled distance from the wall to a virtual detonation point $Z^{\prime}(R)$ can be evaluated by reversing the approach applied for the calculation of $M_{0}$. Gauge G7 is placed at $153 \mathrm{~mm}$ from the wall. Therefore, the scaled distance $Z^{\prime}(G 7)$ from the gauge to the virtual detonation point is obtained by adding the scaled distance from the wall to $Z^{\prime}(R), Z^{\prime}(G 7)=4.199 \mathrm{~m} . \mathrm{kg}^{-1 / 3}$. Eventually, the arrival time of the reflected shock wave at gauge G7 can be estimated using equation (2), $t_{a}=$ $2.683 \mathrm{~ms}$. This value may fit with a reflected peak observed on the pressure history at $2.639 \mathrm{~ms}$. The same approach was then applied to wall EW4 and led to a reflected shock wave arriving at $3.680 \mathrm{~ms}$ at gauge G7. This could match the reflected peak recorded at $3.660 \mathrm{~ms}$.

Therefore, no normal reflection could be responsible for the first reflected peak which should thus come from an oblique reflection. The analysis of a roofless test (Figure 11) revealed that the reflection does not come from the roof but the analytical determination of its origin did not succeed.

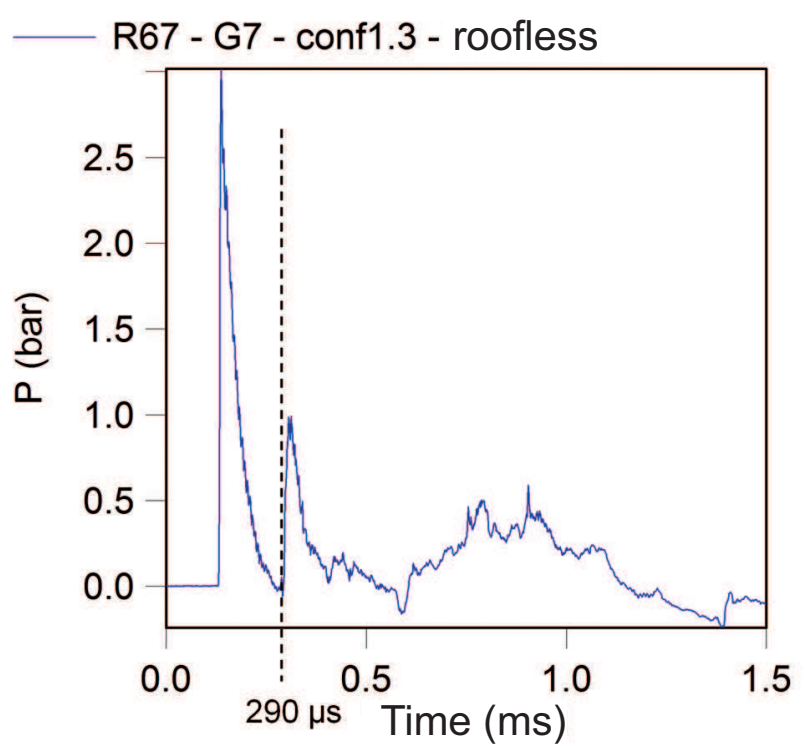

Fig. 11 Pressure history recorded at gauge G7 in configuration 1.3 roofless for a $67 \mathrm{~mm}$ radius gaseous charge

It is also worth noting that the gap between the arrival times of this first reflected peak in semi-confined $(285 \mu \mathrm{s})$ and fully-confined $(290 \mu \mathrm{s})$ is far smaller than for gauge G6. Gauge G7, being placed at the exit of the cell, the detonation products can expand in the corridor and are therefore less confined by the geometry than for gauge G6. The differences in media between the two configurations would then be less significant, accounting for the reduced discrepancy in arrival time.

The pressure histories obtained in confined environment at gauges G6 and G7 is highly complex due to the multiple reflections linked to the enclosed configuration. The fact of being in the cell where the charge is initiated allowed a study of the first reflected peak but it appears difficult to go beyond. It was then decided to further analyze these signals by focusing on some of the 
shock wave parameters (arrival time, incident overpressure, maximal overpressure and total positive impulse).

The scaled arrival time, incident overpressure, maximal overpressure and scaled total positive impulse recorded at gauges G6 and G7 are plotted as a function of the scaled distance in figures 12 and 13.

$$
\overline{t_{a}}\left(\boldsymbol{m s} . k g^{-1 / 3}\right)
$$

(a)

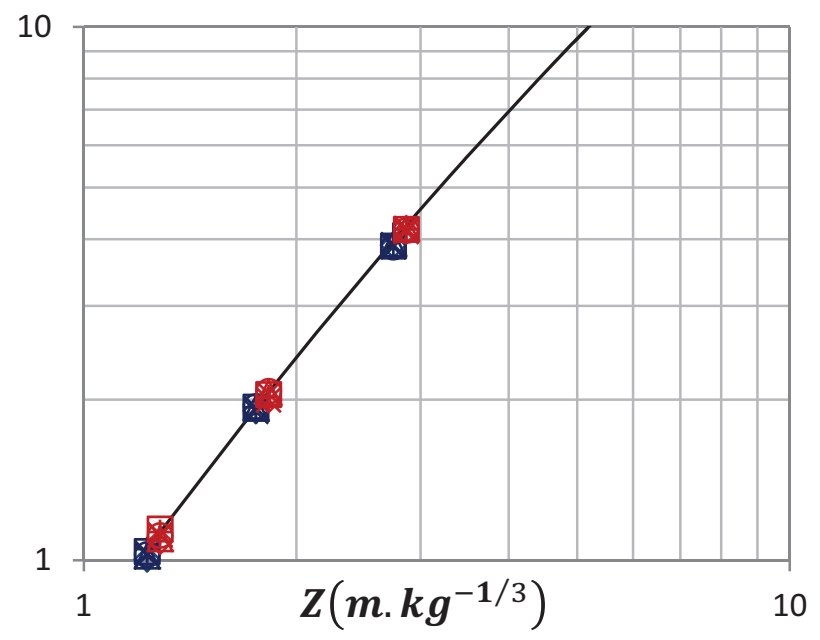

$$
\Delta \boldsymbol{P}_{\boldsymbol{i}}(\text { bar })
$$

(b)

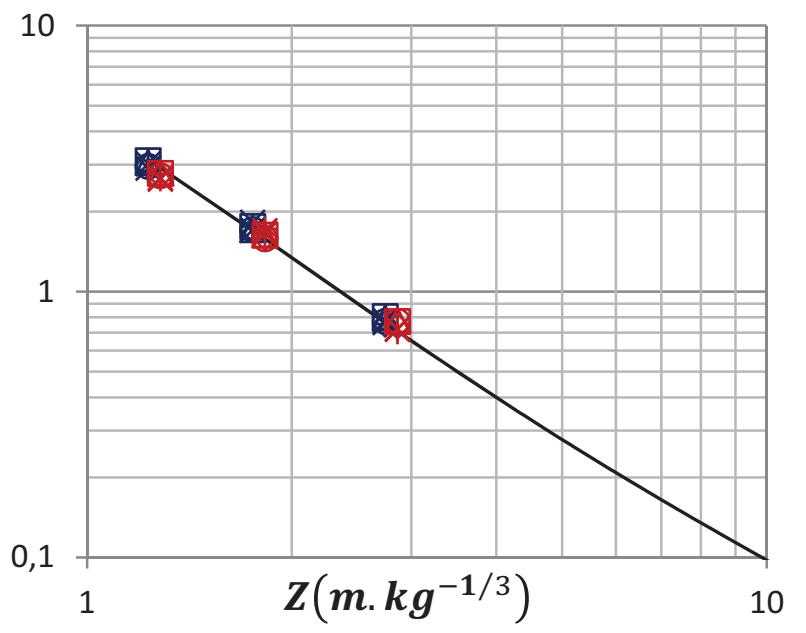

Fig. 12 Evolution of the scaled arrival time (a) and incident overpressure (b) at gauges G6 (blue symbols) and G7 (red symbols) in the model with the scaled distance compared to the free field (configurations $1.1(\diamond), 1.2(\triangle), 1.3(\times), 1.4(\circ)$, $1.5(\square), 2.1(+), 2.2(*)$ and free field $(-))$

For each sensor, the results appear to be centered on three $\mathrm{Z}$ values. These three scaled distances can be directly associated with the three charges evaluated in the experiments (Figures 12 and 13).
$\Delta \boldsymbol{P}_{\max }($ bar $)$

(a)

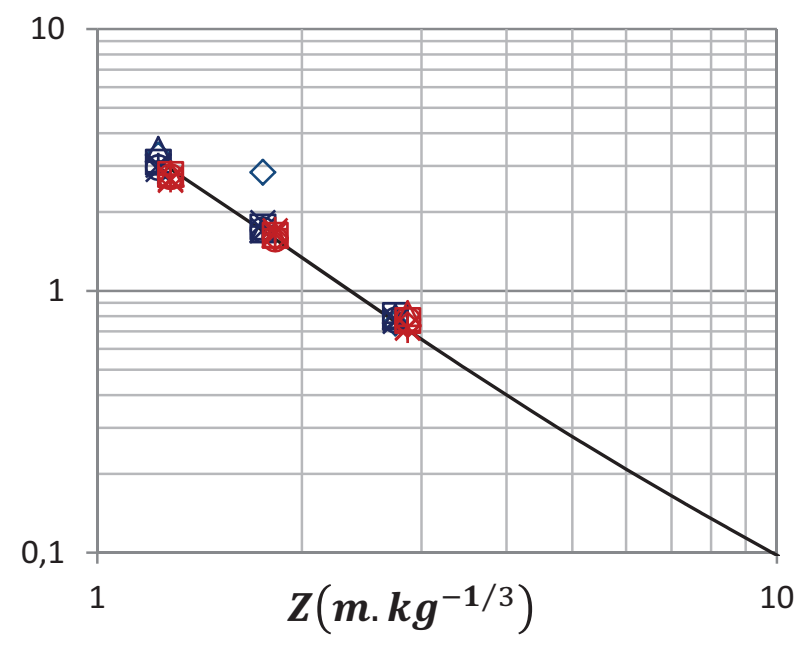

$\overline{\boldsymbol{I}^{+}}\left(\right.$bar. $\left.\boldsymbol{m s} \cdot \boldsymbol{k g}^{-1 / 3}\right)(\mathrm{b})$

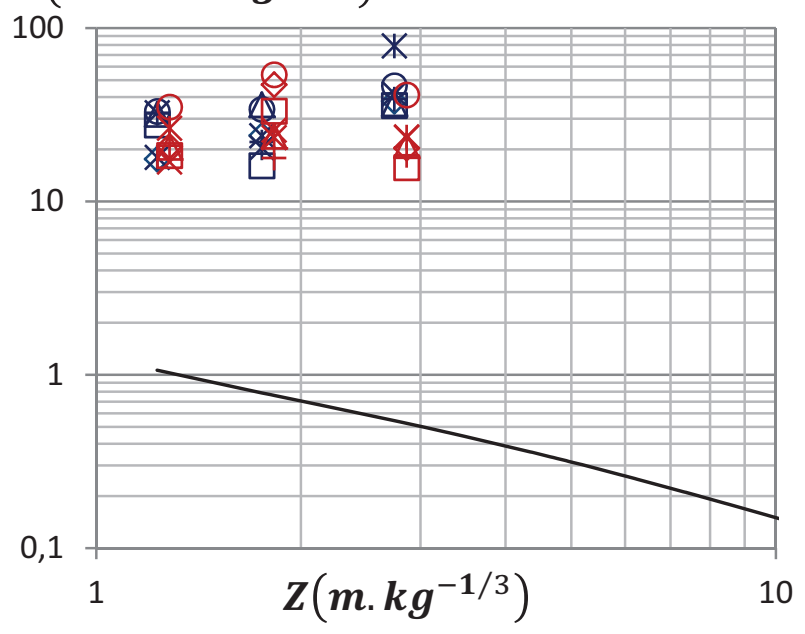

Fig. 13 Evolution of the maximal overpressure (a) and scaled total positive impulse (b) at gauges G6 (blue symbols) and G7 (red symbols) in the model with the scaled distance compared to the free field (configurations $1.1(\diamond), 1.2(\triangle)$ ), $1.3(\times), 1.4(\circ), 1.5(\square), 2.1(+), 2.2(*)$ and free field $(-))$

As was already mentioned, the gauges G6 and G7 are located inside the detonation cell, thus, the incident peak pressure is exactly the same as the one observed in free field. Consequently, the incident overpressure and the arrival time are identical to the free field values (equations (1) and (2)) (Figure 12).

The maximal overpressure observed at gauge G6 corresponds to the incident overpressure except for some points where the former is stronger than the incident overpressure measured in free field (Figure 13-a). These points appear for some specific combination of the volume of the cell and the mass of the charge. More precisely, it happens in configuration 1.1 combined with a $47 \mathrm{~mm}$ radius charge or a $67 \mathrm{~mm}$ radius charge and 
in configuration 1.2 with a $67 \mathrm{~mm}$ radius charge. It is also worth noting that these points are obtained with reflected peaks that can occur after 2 or 13 reflections. Therefore, depending on the volume of the cell and of the mass of the charge, the reflection leading to the maximum overpressure does not originate from the same phenomenon.

However, it is likely that these reflections are the result of the recombination of several shock waves. These results may be explained by the fact that the greater the length of cell 2 , the more important the volume and the more similar the behavior of the shock wave reaching gauge G6 will be to that of a shock wave propagating in free field. The same effect is observed for the mass, since decreasing it shift the behavior of the shock wave reaching gauge G6 toward that of an incident shock wave. This could be the result of the difference in the volume of the detonation products. The volume of the detonation products is indeed directly proportional to the volume of the charge and, as was already mentioned, the geometry of the structure shapes the expansion of the detonation products. Therefore, the stronger the charge, the more significant the differences between free field and confinement regarding the propagation of the shock wave.

The maximal overpressure recorded at gauge G7 follows exactly the reference curve established in free field for the incident overpressure except for one point, where it is slightly stronger (Figure 13-a). This only point, as opposed to the three singular points identified for gauge G6, appears in configuration 1.2 combined with a $30 \mathrm{~mm}$ radius charge and is obtained for the $9^{\text {th }}$ reflected peak. The points of singularity regarding the maximal overpressure do not appear for the same combination of cell and charge volume for the two gauges. This could indicate that the combination tested may not cover the entire range of maximal overpressure that could be encountered for these two pressure transducers. This will have to be taken into account when these data will be used to draw an evolution law for the maximal overpressure.

The maximal positive impulse is far greater than the one in free field due to the multiple reflections that occur in a confined environment. However, no law describing the evolution of the maximal positive impulse with the volume could be found for this cell (Figure $13-\mathrm{b})$.

\subsection{Gauges G8}

Gauge G8, although not located inside cell 2, is also in direct view of the charge, setting it apart from the other pressure transducers in the corridor. The pressure histories recorded at gauge G8 in free field and in configuration 1.3 are reported in figures 14 and 15 .

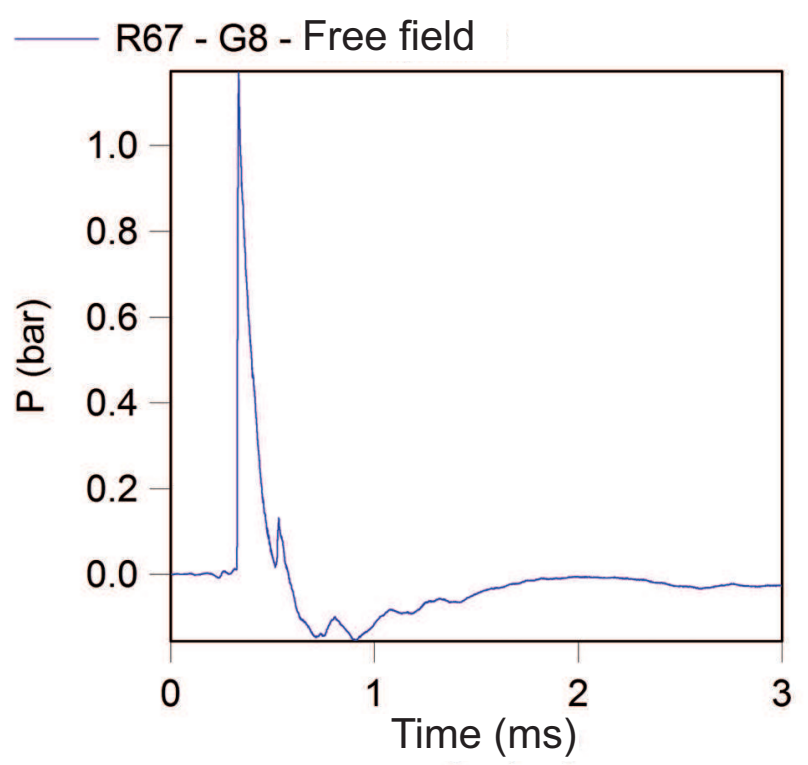

Fig. 14 Pressure history recorded at gauge G8 in free field for a $67 \mathrm{~mm}$ radius gaseous charge

Since the sensor is in direct view of the load, the incident peak observed in free field can also be found on the pressure history recorded in a confined environment. The first reflected peak appears at $491 \mu$ s (Figure 14). It is also found in semi-confined experiments and consequently is not due to the roof (Figure 15).

The analytical study of the oblique reflection on walls IW1 and IW2 was carried out exactly as for gauge G6. It reveals that two reflected shock waves arrive simultaneously from the two walls at gauge G8 at $802 \mu \mathrm{s}$. These reflections don't match the first reflected peak but could correspond to the peak observed at $756 \mu \mathrm{s}$. As for gauge G6, the time difference could be explained by the hypothesis on the calculations. Similarly to gauge G7, Mach stems are propagating on walls IW1 and IW2 toward gauge G8. Yet, they will be diffracted by the edges of the walls before reaching gauge G8. Once again, two normal reflections, from walls EW2 and EW4, hit gauge G8. Knowing the distances between gauge G8 and the two walls, the approach used for gauge G7 can be applied to estimate the arrival time of these normal reflections. This leads to an arrival time of $1.833 \mathrm{~ms}$ 


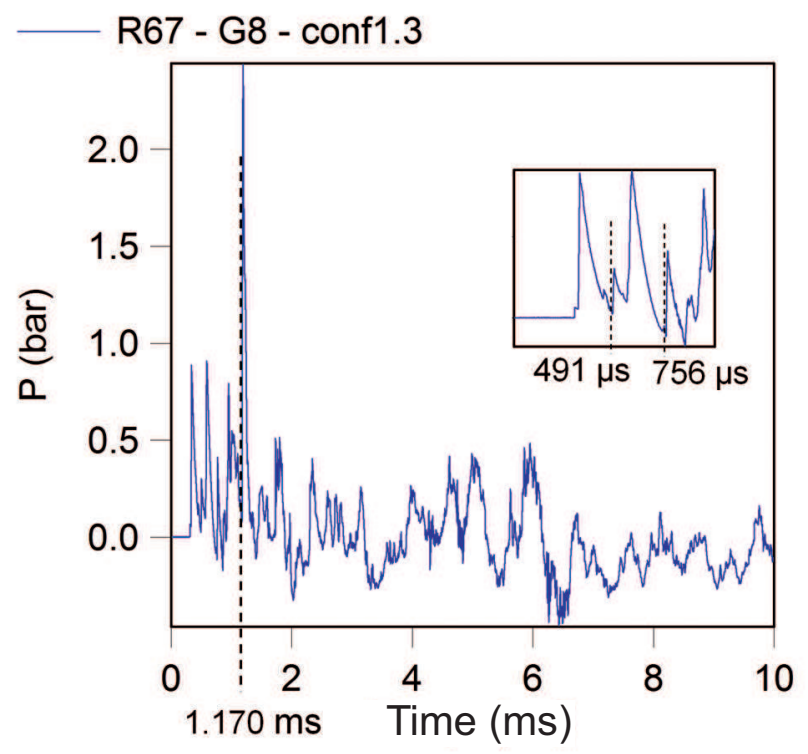

Fig. 15 Pressure history recorded at gauge G8 in configuration 1.3 for a $67 \mathrm{~mm}$ radius gaseous charge

for the reflection on wall EW2 and $4.602 \mathrm{~ms}$ on wall EW4. Thus, the analytical approach used in this study does not enable us to determine the origin of the first reflected peak.

The main difference with gauges G6 and G7 is related to the maximum peak pressure. Indeed, while the maximum pressure was generally associated with the incident shock wave for gauges G6 and G7, it is always obtained for a reflection at gauge G8. Moreover, the maximum overpressure is greatly increased (50\%) compared to the free field. In an attempt to determine the origin of this peak, a comparison with a roofless test was carried out (Figure 16). The maximum peak pressure observed at $1.170 \mathrm{~ms}$ in configuration 1.3 does not appear in the semi-confined environment. This reflection is therefore linked to the presence of the roof. This phenomenon has been observed for all volumes and charges studied.

In order to investigate the effect of the change in volume on the parameters of the shock wave recorded at gauge G8, the scaled arrival time, the incident overpressure, the maximal overpressure and the scaled total positive impulse have been plotted as a function of the scaled distances for all volumes and charges evaluated (Figures 17 and 18).

It was previously shown during the analysis of the pressure history that the incident peak pressure was identical to the one observed in free field. This can also logically be found when studying the values of the incident shock wave arrival time and of the incident over-

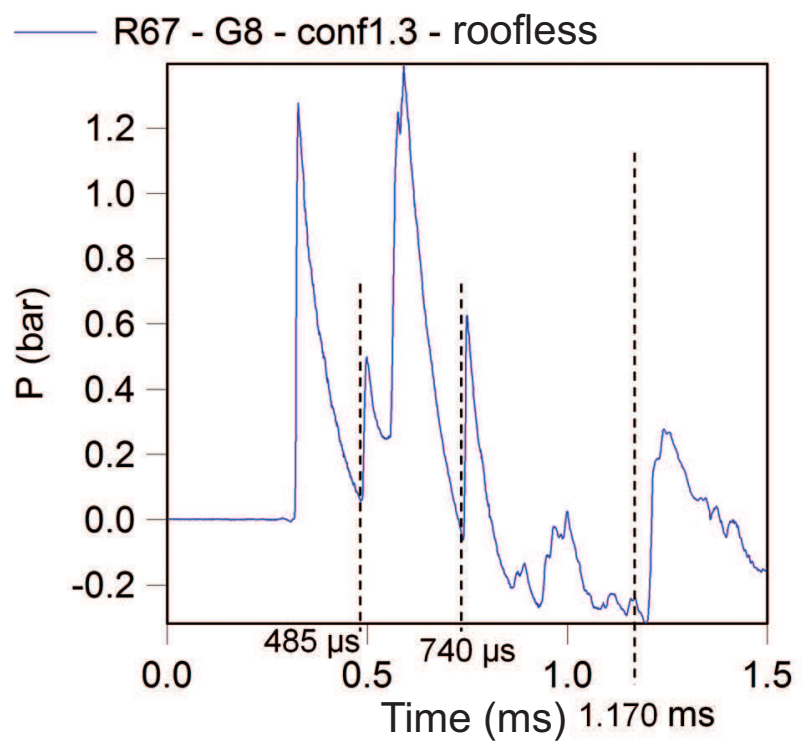

Fig. 16 Pressure history recorded at gauge G8 in configuration 1.3 roofless for a $67 \mathrm{~mm}$ radius gaseous charge

pressure. These fit very well the reference curves established in free field (equations (1) and (2)) (Figure 17). Figure 18-a confirms what was observed while analyzing the pressure history. That is to say that the maximal overpressure observed at gauge G8 never fit the free field value. Moreover, unlike the case of gauges G6 and G7, no correlation with the scaled distance was found for the maximal overpressure at gauge G8.

Once again, the total positive impulse is greatly increased when compared to the free field level due to the multiple reflections that occur in a confined environment. No evolution law for this parameter with the volume of the cell could be established (Figure 18-b). 


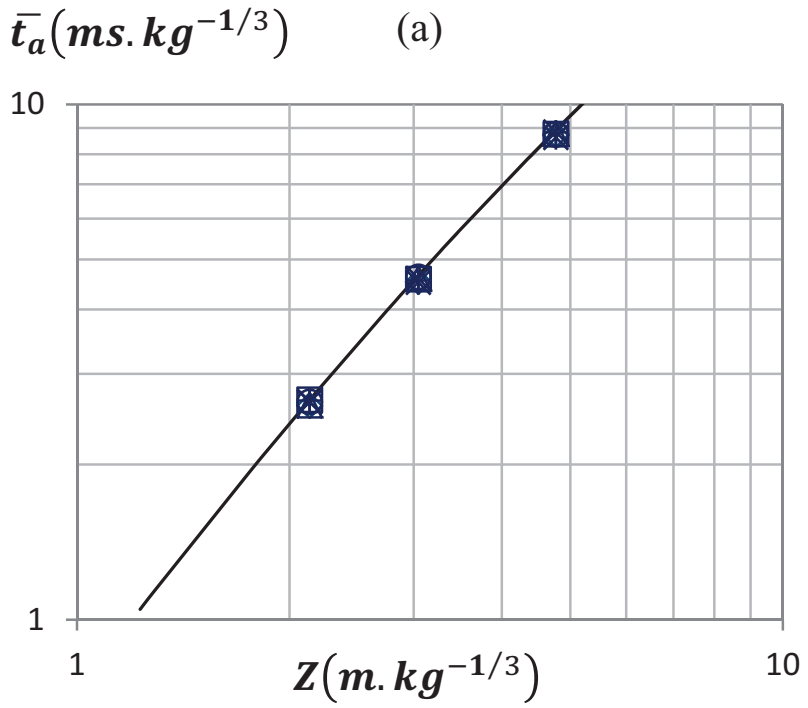

$\Delta \boldsymbol{P}_{i}($ bar $)$

(b)

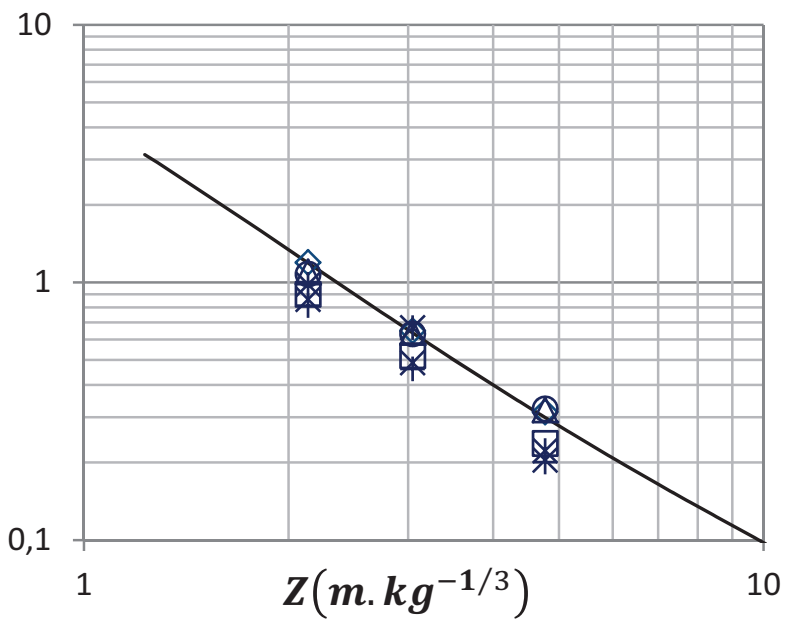

Fig. 17 Evolution of the scaled arrival time (a) and incident overpressure (b) at gauge G8 in the model with the scaled distance compared to the free field

\section{Phenomenology}

Unlike what was observed in the detonation cell, the sensors placed in the other cells of the model are shielded from the charge by the structure. They are therefore hit by a wave that has interacted with several obstacles. The analysis of their pressure history is thus more complex. Yet, the model has some geometrical properties that can be used to study the pressure histories of some pressure transducers (Figure 2).

$$
\Delta P_{\text {max }}(\text { bar })
$$

(a)

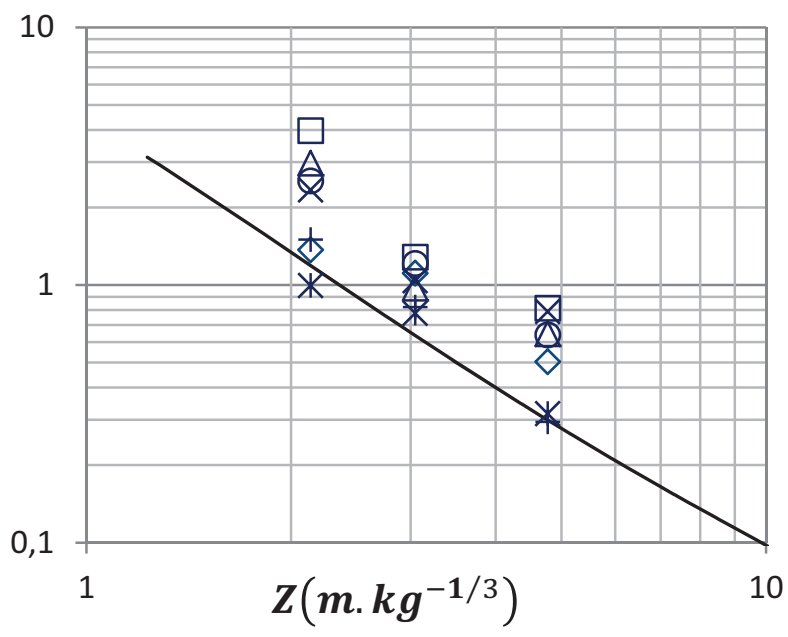

$\overline{I^{+}}\left(\text {bar. } m s \cdot \boldsymbol{k g}^{-1 / 3}\right)^{(\mathrm{b})}$

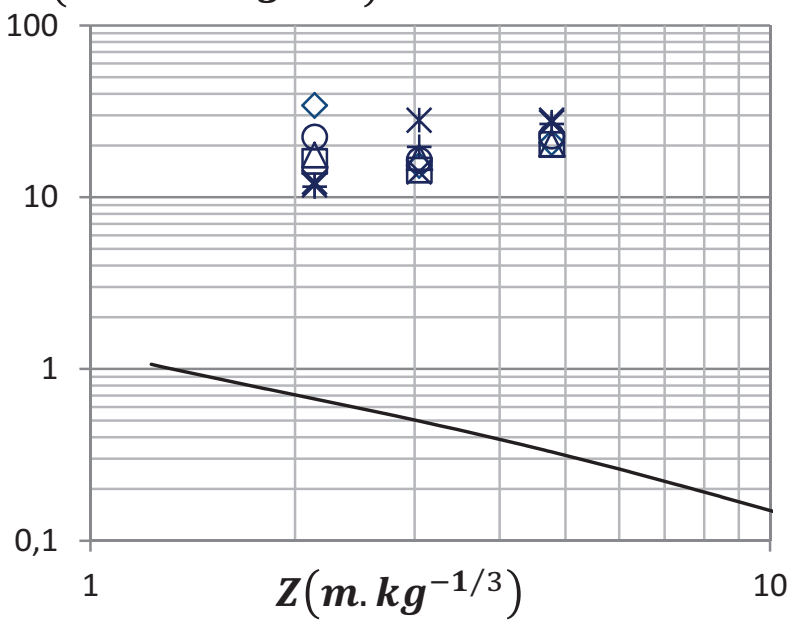

Fig. 18 Evolution of the maximal overpressure (a) and scaled total positive impulse (b) at gauge G8 in the model with the scaled distance compared to the free field

\subsection{Similarity of cells 1 and 3}

Cells 1 and 3, placed on both sides of the detonation cell, present some similarities (Figure 2). Indeed, gauges G2 and G9, G1 and G10, and G3 and G11 are three couples that are in symmetrical positions relatively to the charge in configuration 1 . In configuration 2 , only gauges G2 and G9 remain in such a configuration. Nevertheless, these two cells have a main difference since the corridor is closed by wall EW1 in cell 1 whereas it passes by cell 3 toward cell 4 . 


\subsubsection{Gauges G2 and G9}

Gauges G2 and G9 are both placed in the corner formed by the back wall (EW4) and the side wall closest to the charge in their respective cells (Figure 2). The pressure histories recorded at gauges G2 and G9 in configuration 1.3 are plotted on the same graph (Figure 19).

The incident shock wave reaching gauges G2 and G9

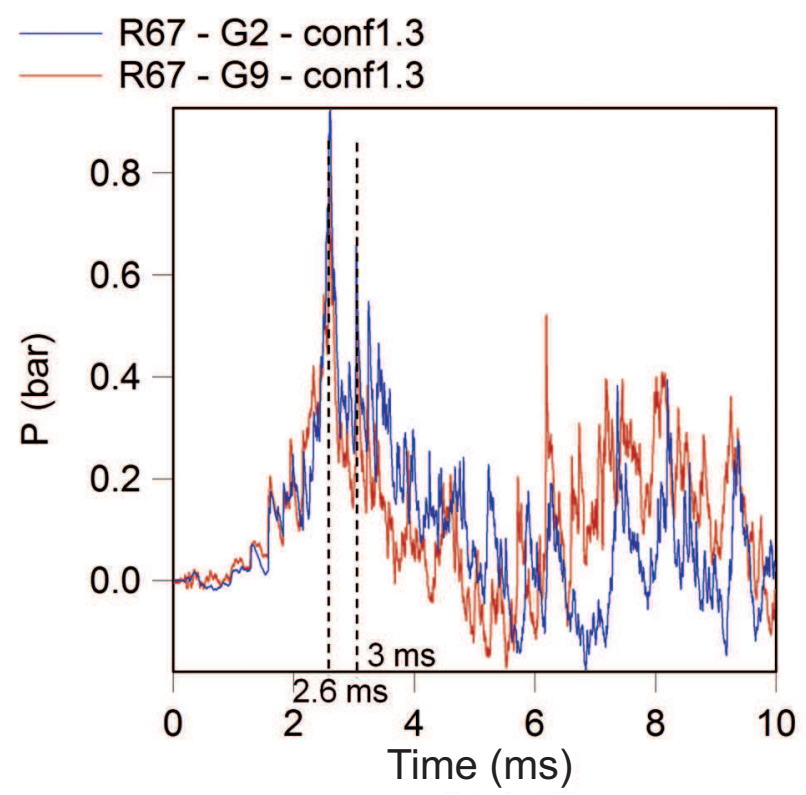

Fig. 19 Pressure histories recorded at gauges G2 and G9 in configuration 1.3 for a $67 \mathrm{~mm}$ radius gaseous charge

does not present the same shape as in free field or in cell 2 (Figure 19). This is due to the interaction of the shock wave with the obstacles on its way to the sensors. Looking at both signals, it appears that the evolution pattern of the pressure is very similar up to $3 \mathrm{~ms}$ with very close arrival times of the incident shock waves and maximal overpressures (mean gap of $0.7 \%$ for the arrival times and $14 \%$ for the maximal overpressures). From this point, differences in amplitudes can be observed and seem to increase with time. Therefore, it seems that, in the first few moments $(3 \mathrm{~ms})$, the waves reaching gauges G2 and G9 are subjected to the same phenomenon.

The pressure histories at gauges G2 and G9 were also recorded in semi-confined environment, that is to say roofless, in configuration 1.3 (Figure 20). It is noteworthy, that without the roof, the waves can get around the top of the walls. The shortest path of the shock wave can thus diverge from that in fully enclosed system. This may lead to time discrepancies between the arrival times in semi-confined and confined environment and alter the pressure waves that eventually reach the

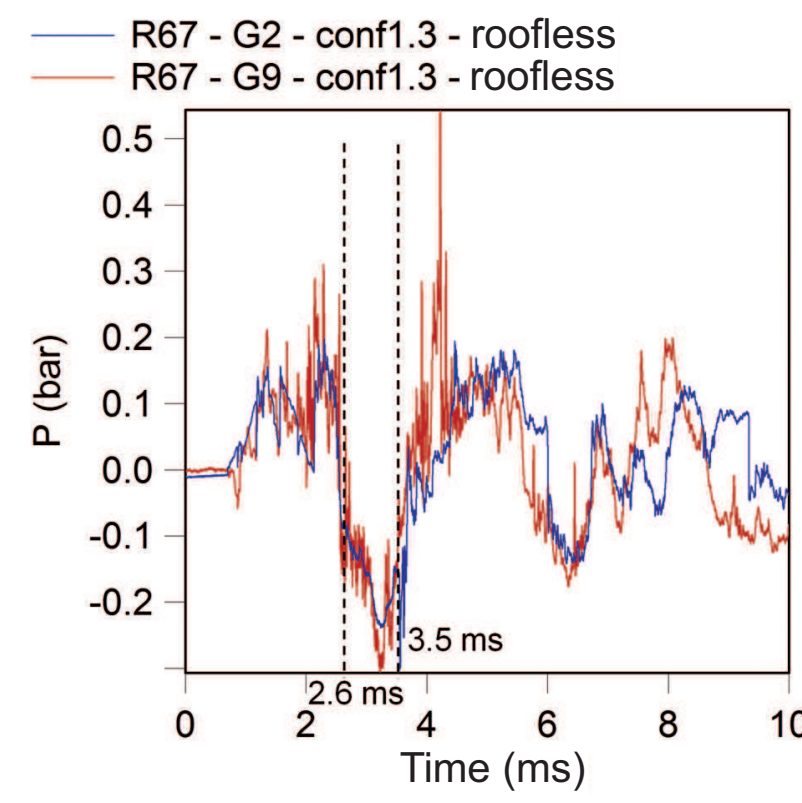

Fig. 20 Pressure histories recorded at gauges G2 and G9 in configuration 1.3 roofless for a $67 \mathrm{~mm}$ radius gaseous charge

transducer. Yet, the similarity of the evolution of the pressure histories recorded at gauges G2 and G9 remains in roofless configuration. Nevertheless, the maximal overpressure peak observed at $2.6 \mathrm{~ms}$ in configuration 1.3 disappears in the roofless test. The maximal overpressure is then linked to the roof. Moreover, the pressure histories keep the same shape longer (up to $3.5 \mathrm{~ms}$ ). The roof seems to strengthen the effect of wall EW1 on the shock wave propagation.

In configuration 2.1, the differences between the two pressure histories occur after $2 \mathrm{~ms}$. Only the two first peaks are similar (Figure 21). This can probably be explained by the width of the cells that are different for cells 1 and 3 in this configuration.

\subsubsection{Gauges G3 and G11}

Gauges G3 and G11 are placed at the exit of cells 1 and 3 and are also in an almost symmetrical position relatively to the charge in configuration 1 (Figure 2). Figure 22 shows the pressure histories recorded at gauges G3 and G11 in configuration 1.3.

In this case, the shape of the incident shock wave is closer to one that could be observed in free field (Figure 22). The pressure histories recorded at gauges G3 and G11 follow almost the same evolution until $2 \mathrm{~ms}$ (mean gap of $0.5 \%$ in the reflected peaks arrival times). Unlike what was found for gauges G2 and G9, the maximum overpressures are associated with the same peak (2.083 ms for G3 and $1.452 \mathrm{~ms}$ for G11). 


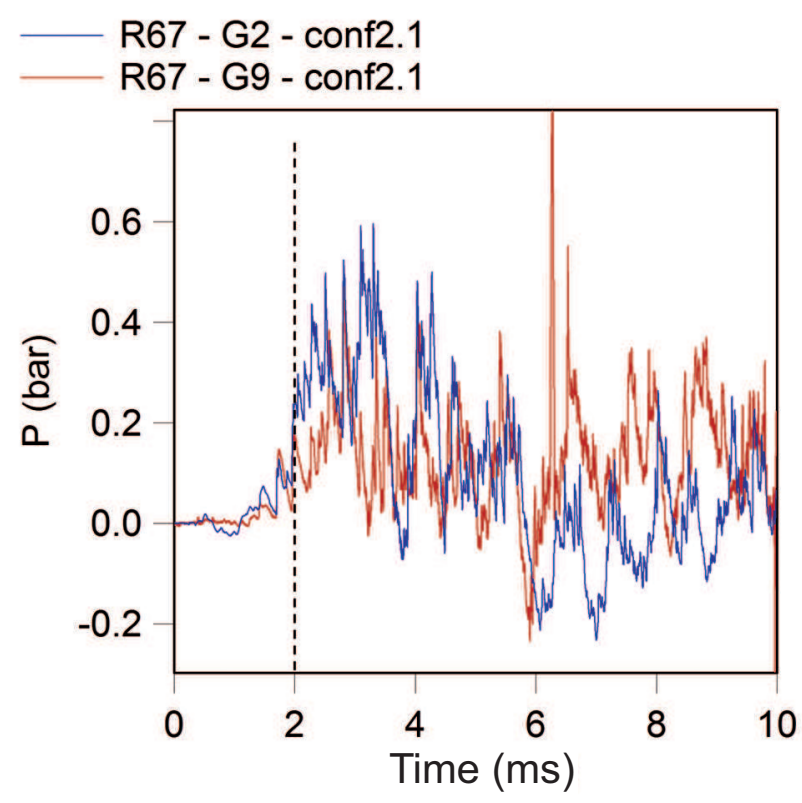

Fig. 21 Pressure histories recorded at gauges G2 and G9 in configuration 2.1 for a $67 \mathrm{~mm}$ radius gaseous charge

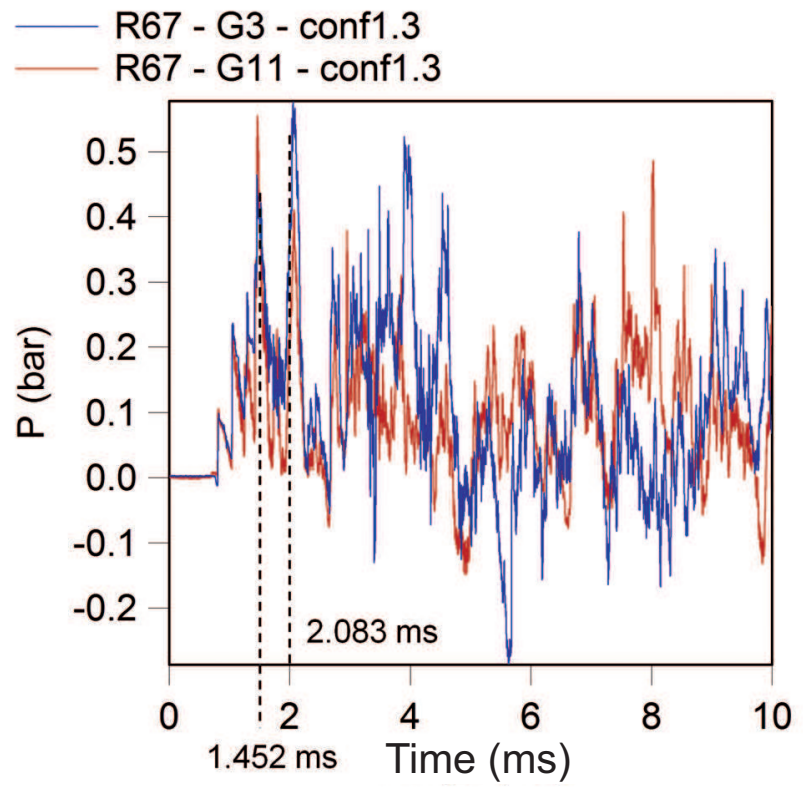

Fig. 22 Pressure histories recorded at gauges G3 and G11 in configuration 1.3 for a $67 \mathrm{~mm}$ radius gaseous charge

However, the arrival time of the incident shock wave is identical $(0.07 \%$ gap) as well as the incident overpressure (1\% gap). Gauge G3 is closer to wall EW1 than G2 which could be the reason why the discrepancies between gauges G3 and G11 happen sooner than for gauges G2 and G9.

This observation seems to be confirmed by the roofless tests (Figure 23). Indeed, as in the fully confined case, the differences between G3 and G11 appear after $2 \mathrm{~ms}$.

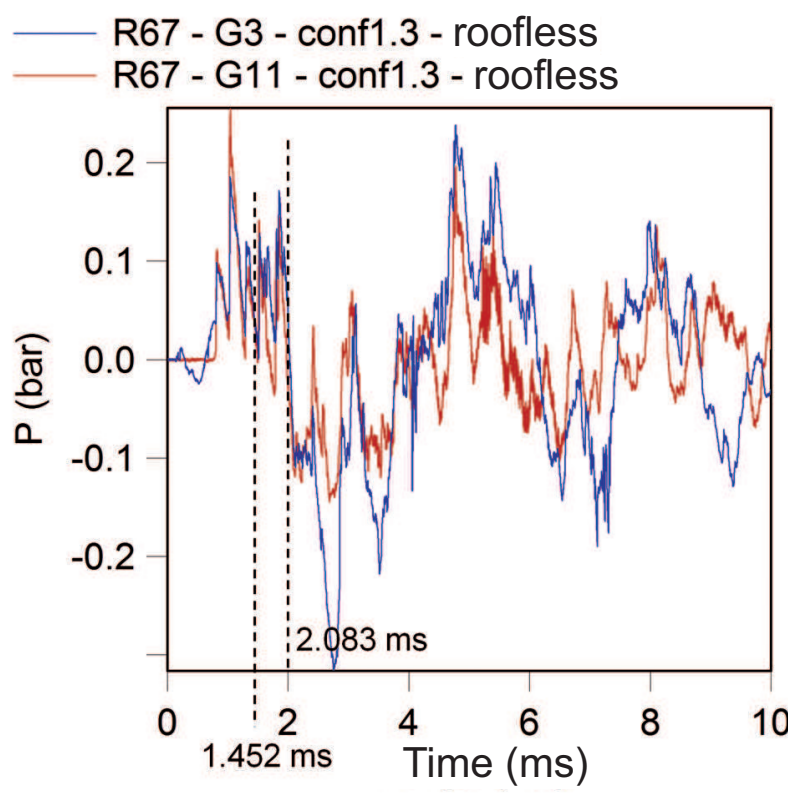

Fig. 23 Pressure histories recorded at gauges G3 and G11 in configuration 1.3 roofless for a $67 \mathrm{~mm}$ radius gaseous charge

Figure 23 also reveals that the maximum overpressure at gauges G3 and G11 is linked to the roof as the peaks found in configuration 1.3 disappear in roofless conditions. Yet, the difference in the arrival time associated with the maximal overpressure peak indicates that the origins are not the same.

In configuration 2, gauges G3 and G11 loose their similarity. The distances from the sensors to the center of the charge are no more alike. Thus, no similar evolution can be found in the pressure histories in this configuration. This is perfectly illustrated by figure 24 .

\subsubsection{Gauges G1 and G10}

To conclude the study of the similarity of cells 1 and 3 , the behavior of gauges G1 and G10 are analyzed. They are both in the corner formed by wall EW4 and by the wall the farthest from the charge in their respective cells (EW1 and EW3). The pressure histories recorded at gauges G1 and G10 in configuration 1.3 are plotted in figure 25 .

Although a similar evolution of the pressure histories can be observed in the first few moment $(0.5 \%$ gap in the reflected peaks arrival times), the amplitudes present some difference from the beginning. More precisely, the incident peaks have only the arrival time in common, unlike what was found for two other couples studied previously. Nevertheless, this could be due to the quality of the beginning of the signals with para- 


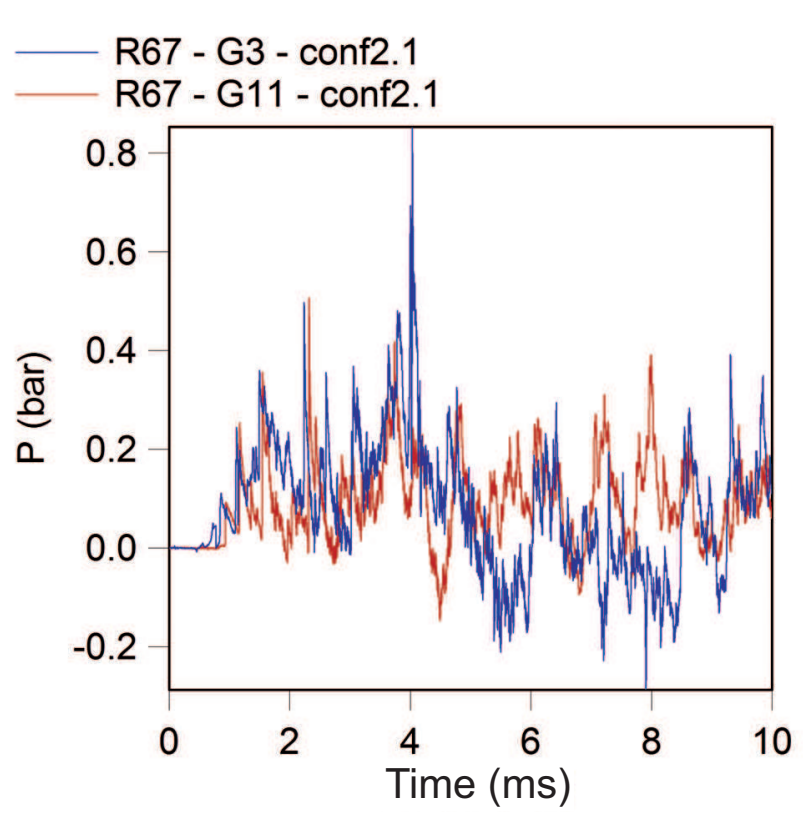

Fig. 24 Pressure histories recorded at gauges G3 and G11 in configuration 2.1 for a $67 \mathrm{~mm}$ radius gaseous charge

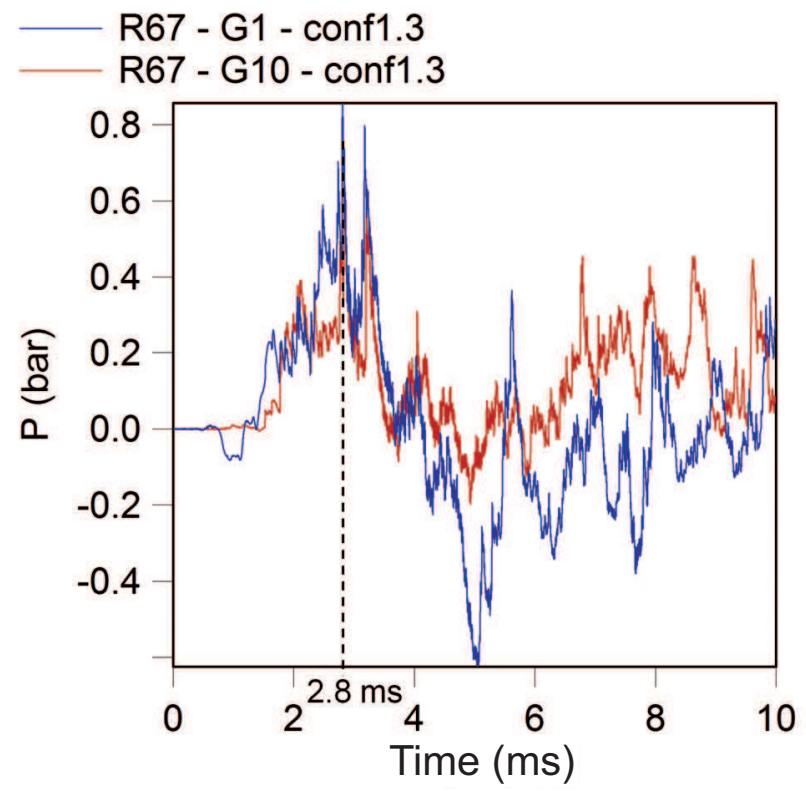

Fig. 25 Pressure histories recorded at gauges G1 and G10 in configuration 1.3 for a $67 \mathrm{~mm}$ radius gaseous charge

sites altering the incident shock wave. A more detailed analysis of this parasites is presented in a paragraph hereafter(cf. 5.2).

The reflected peaks leading to the maximal overpressure occurs at almost the same time for both sensors (2.80 $\mathrm{ms}$ and $2.83 \mathrm{~ms}$ ). It seems then that the same phenomenon is responsible for the maximal overpressure for gauges G1 and G10 as was the case for gauges G2 and G9.
Figure 26 describe the path that the wave has to travel to reach gauges G1 and G10. It shows that two diffractions are required which explained the difference of the shape of the incident shock wave with the free field.

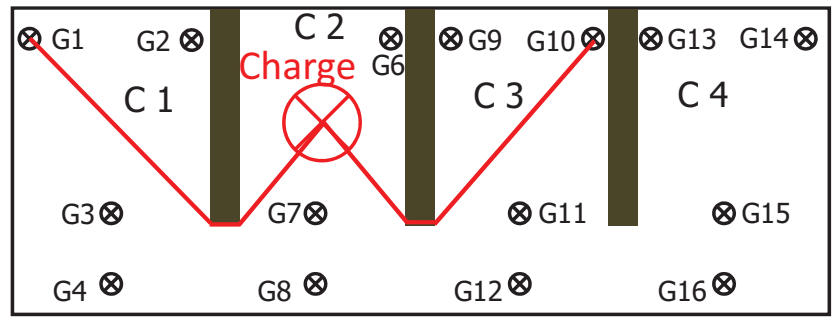

Fig. 26 Shock wave path to gauges G1 and G10

Figure 27 presents the pressure histories recorded at gauges G1 and G10 in semi-confined environment. It appears that the maximum overpressure is linked to the roof since the maximum overpressure peaks observed at $2.8 \mathrm{~ms}$ can not be found in roofless tests. Moreover, the pressure histories have the same evolution pattern in the first $10 \mathrm{~ms}$ ( $2 \%$ mean gap in the arrival times). Therefore, the roof seems to reinforce the differences between gauges G1 and G10 and thus the effect of wall EW1.

As gauges G3 and G11, gauges G1 and G10 are no more in symmetrical position in configuration 2. Consequently, there is no need to study the symmetry of

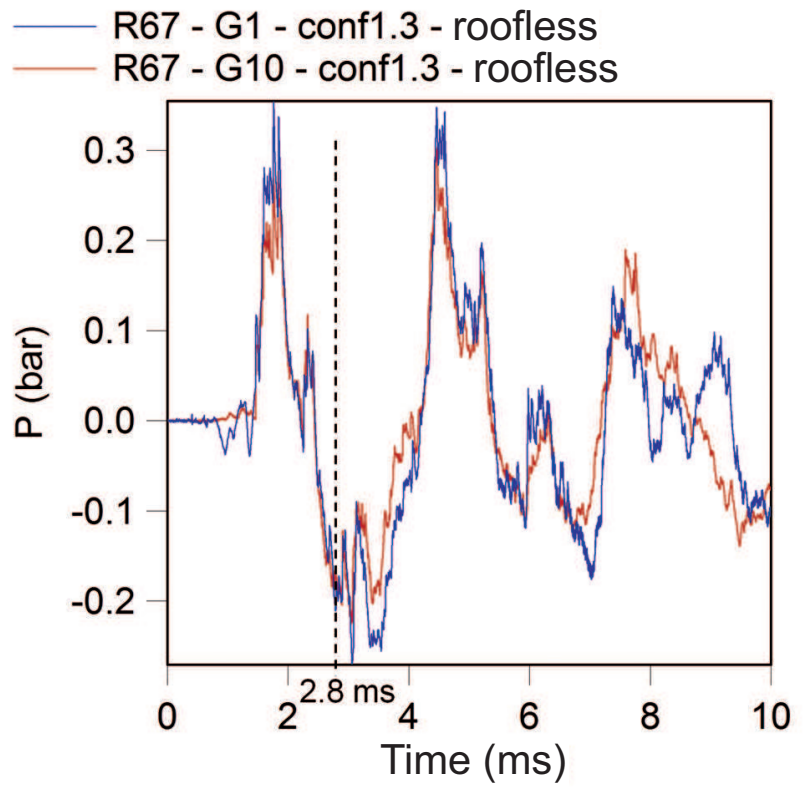

Fig. 27 Pressure histories recorded at gauges G1 and G10 in configuration 1.3 roofless for a $67 \mathrm{~mm}$ radius gaseous charge 


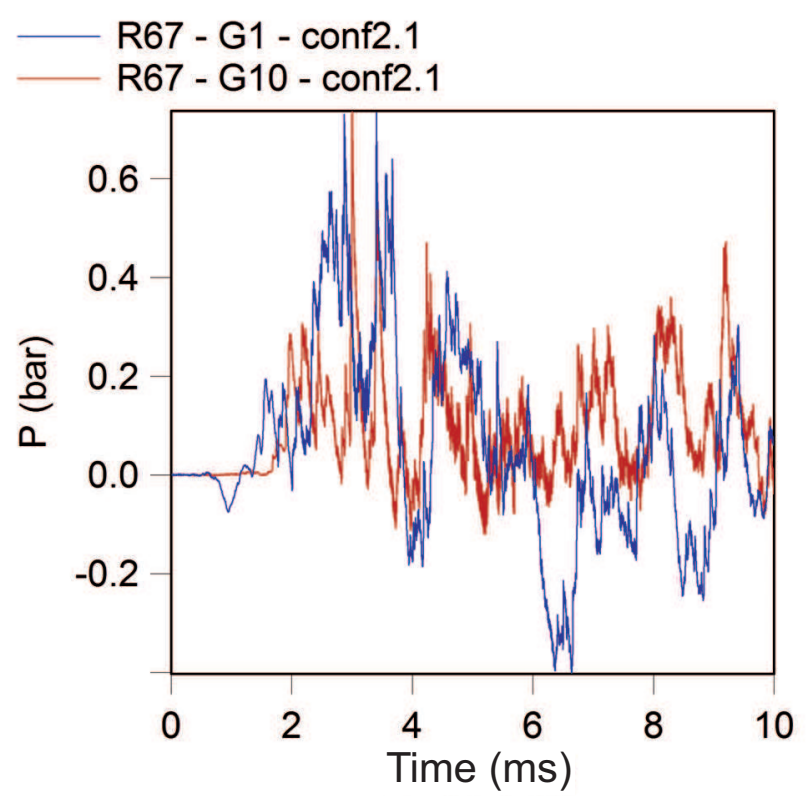

Fig. 28 Pressure histories recorded at gauges G1 and G10 in configuration 2.1 for a $67 \mathrm{~mm}$ radius gaseous charge

these two pressure transducers (Figure 28).

Finally, cells 1 and 3 present similarity regarding the evolution of the pressure histories in the first moments, except for gauges G1 and G10. Then, the differences due to the wall closing cell 1 (EW1) appear. The singular case of gauges G1 and G10 may be explained by the fact that these two sensors are placed along walls of different length and are thus subjected to different reflections.

\subsection{Comparison of cells 1 and 4}

Cells 1 and 4 have also some similarity as these two cells are the extreme cells of the model (Figure 2). Moreover, the volume of both cells is always identical no matter the configuration.

Gauges G1 and G14 are respectively located in the corners formed by the side walls (EW1 and EW3) and wall EW4 in cell 1 and cell 4 (Figure 2). These two gauges are at both extremities of the model but at a different distance from the charge and have the peculiarity of always keeping the same position in all evaluated configurations.

The pressure histories at gauges G1 and G14 have been plotted in figure 29.

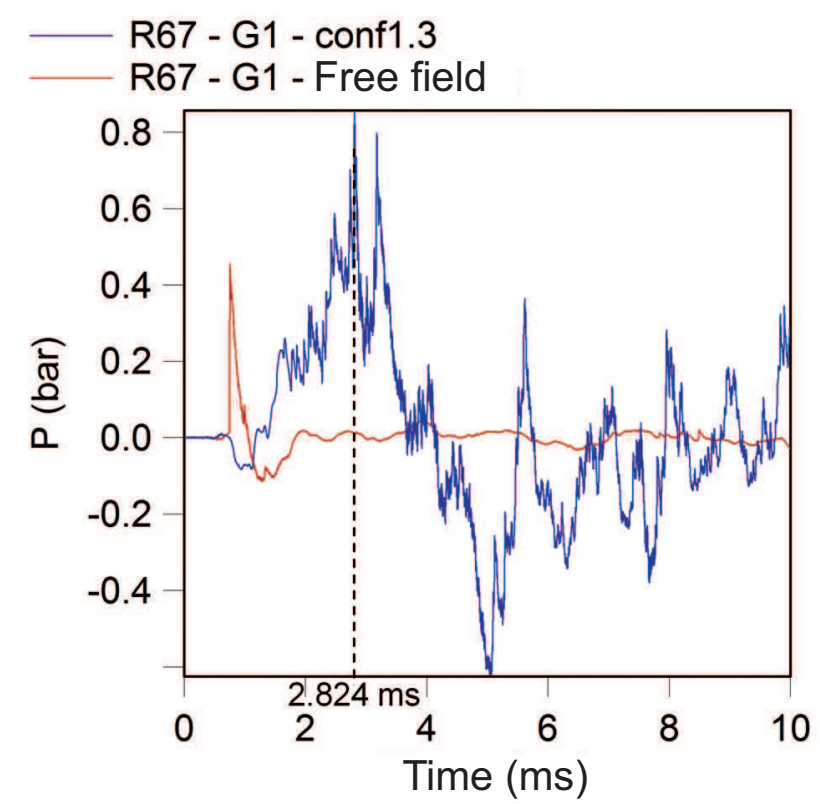

R67 - G14 - conf1.3

R67 - G14 - Free field

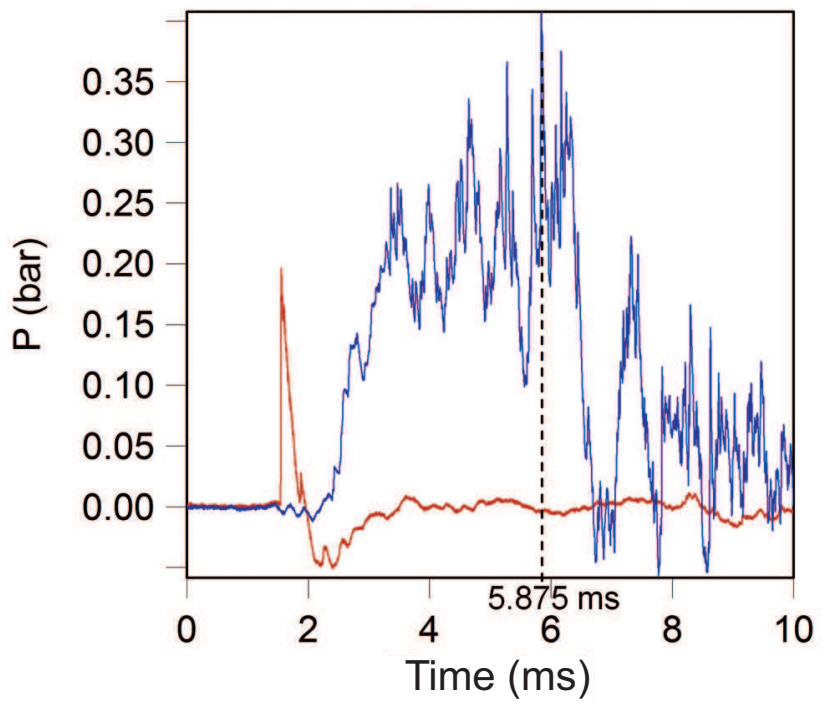

Fig. 29 Pressure histories recorded at gauges G1 and G14 in configuration 1.3 and in free field for a $67 \mathrm{~mm}$ radius gaseous charge

The discrepancy in the arrival time of the incident shock wave is due to the differences between the distances the wave has to travel to reach the two sensors, gauge G1 being much closer to the charge (688 mm) than gauge G14 (1003 mm). Consequently, the incident overpressure recorded at these gauges is weaker than in free field. The structure of the building forms obstacles to the shock wave propagation that reduces the pressure levels of the incident shock wave. Yet, it appears that the maximal overpressure is greatly increased compared to the free field for both gauges G1 (84\%) and G14 
$(100 \%)$. The confinement generates multiple reflections that by interacting with each other give, in this configuration, higher maximal overpressures than in free field. Thus, although the pressure transducers are located in positions that seem protected against the charge, the blast in configuration 1.3 is stronger than in free field.

Roofless tests have also been carried out in this configuration. The pressure histories recorded at gauge G1 and G14 are plotted on the same graph (Figure 30). This graph shows that the maximal overpressure in a confined environment depends on the presence of the roof for both gauges G1 and G14. Moreover, the maximal overpressure in a semi-confined environment is on average $26 \%$ weaker than the incident overpressure recorded in free field. The roofless conditions seem to prevent the loss of the protective effect due to distance from the charge observed in confined environment.

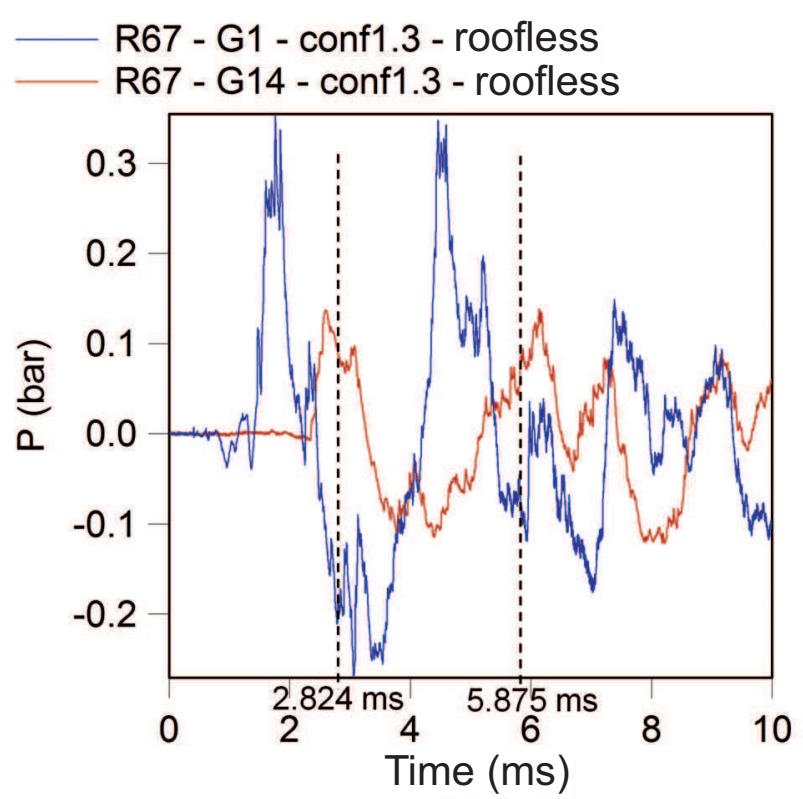

Fig. 30 Pressure histories recorded at gauges G1 and G14 in configuration 1.3 roofless for a $67 \mathrm{~mm}$ radius gaseous charge

The trend observed in configuration 1.3 also appears in the whole configuration 1 (Tables 3 and 4).

These tables show that with the exception of the $30 \mathrm{~mm}$ radius charge and in most cases, the increase in the maximal overpressure compared to the free field is more critical at gauge G14 ( $86 \%$ on average) than at gauge G1 ( $62 \%$ on average). The increase in the total positive impulse is, in turn, always stronger for gauge G14 (by a factor of 71) than for gauge G1 (by a factor of 53). Thus, the protective effect due to the longer distance
Table 3 Differences between incident and maximal overpressures in configuration 1 and in free field at gauges G1 and G14

\begin{tabular}{|c|c|c|c|}
\hline \multirow{3}{*}{ Configuration } & $\begin{array}{c}\text { Charge } \\
\text { radius } \\
(\mathrm{mm})\end{array}$ & $\frac{\Delta P_{\max }}{\Delta P_{\text {free }}}(G 1)$ & $\frac{\Delta P_{\max }}{\Delta P_{\text {free }}}(G 14)$ \\
\hline \multirow{3}{*}{1.1} & 30 & 2.212 & 1.669 \\
\cline { 2 - 4 } & 47 & 1.609 & 1.938 \\
\cline { 2 - 4 } & 67 & 1.831 & 1.941 \\
\hline \multirow{3}{*}{1.2} & 30 & 1.339 & 1.697 \\
\cline { 2 - 4 } & 47 & 1.318 & 1.916 \\
\cline { 2 - 4 } & 67 & 1.500 & 1.893 \\
\hline \multirow{3}{*}{1.3} & 30 & 1.424 & 1.321 \\
\cline { 2 - 4 } & 47 & 1.286 & 1.719 \\
\cline { 2 - 4 } & 67 & 1.844 & 2.008 \\
\cline { 2 - 4 } & 30 & 1.462 & 1.432 \\
\cline { 2 - 4 } & 47 & 1.283 & 1.589 \\
\hline \multirow{3}{*}{1.5} & 37 & 1.902 & 2.265 \\
\cline { 2 - 4 } & 30 & 1.570 & 1.278 \\
\cline { 2 - 4 } & 67 & 1.251 & 1.574 \\
\hline & & 2.404 & 1.788 \\
\hline
\end{tabular}

Table 4 Differences between scaled total positive impulse in configuration 1 and in free field at gauges G1 and G14

\begin{tabular}{|c|c|c|c|}
\hline Configuration & $\begin{array}{c}\text { Charge } \\
\text { radius } \\
(\mathrm{mm})\end{array}$ & $\frac{I^{+}{ }_{t o t}}{I^{+}{ }_{i}}(G 1)$ & $\frac{I^{+}{ }_{t o t}}{I^{+}{ }_{i}}(G 14)$ \\
\hline \multirow{3}{*}{1.1} & 30 & 127 & 175 \\
\hline & 47 & 76 & 97 \\
\hline & 67 & $\overline{46}$ & 55 \\
\hline \multirow{3}{*}{1.2} & 30 & 101 & 168 \\
\hline & 47 & 69 & 109 \\
\hline & 67 & 44 & 61 \\
\hline \multirow{3}{*}{1.3} & 30 & $\overline{94}$ & 122 \\
\hline & 47 & 64 & 92 \\
\hline & 67 & 44 & 54 \\
\hline \multirow{3}{*}{1.4} & 30 & $\overline{94}$ & 124 \\
\hline & 47 & 61 & 82 \\
\hline & 67 & 43 & 51 \\
\hline \multirow{3}{*}{1.5} & 30 & 99 & 113 \\
\hline & 47 & 53 & 65 \\
\hline & 67 & 34 & 45 \\
\hline
\end{tabular}

from the charge is attenuated by the confinement.

As was previously found in configuration 1.3, a significant increase in the maximal overpressure compared to the free field is observed at gauges G1 (61\%) and G14 (140\% in configuration 2.1) (Figure 31). The change in volume of cells 1 and 4 does not seem to alter the phenomenology that had been observed in configuration 1 . This is confirmed by figure 32 that plots the pressure histories recorded at gauges G1 and G14 in configuration 2.1 roofless. Indeed, as was found in configuration 1.3, the maximal overpressure recorded at gauges G1 and G14 in confined environment is due to the roof. In addition, the maximal overpressure in a semi-confined environment is on the same order of magnitude $(0.5 \%$ 
gap on average) than the incident overpressure obtained in free field.

\section{R67 - G1 - conf2.1}

R67 - G1 - Free field

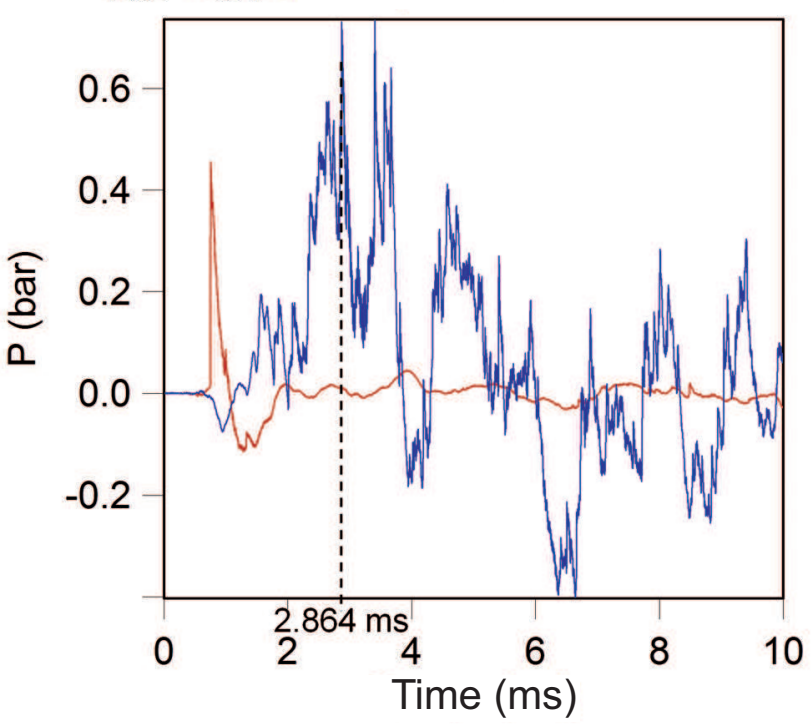

R67 - G14 - conf2.1

R67 - G14 - Free field

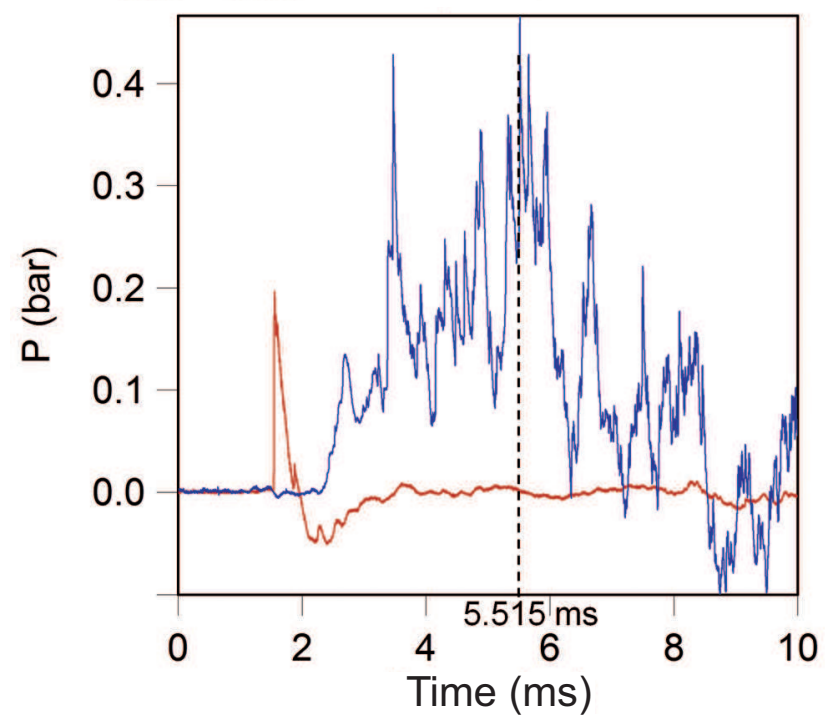

Fig. 31 Pressure histories recorded at gauges G1 and G14 in configuration 2.1 and in free field for a $67 \mathrm{~mm}$ radius gaseous charge

Tables 5 and 6 present the numerical values of the differences between the maximal overpressure and total positive impulses obtained in configuration 2.1 and in free field.
R67 - G1 - conf2.1 - roofless

R67 - G14 - conf2.1 - roofless

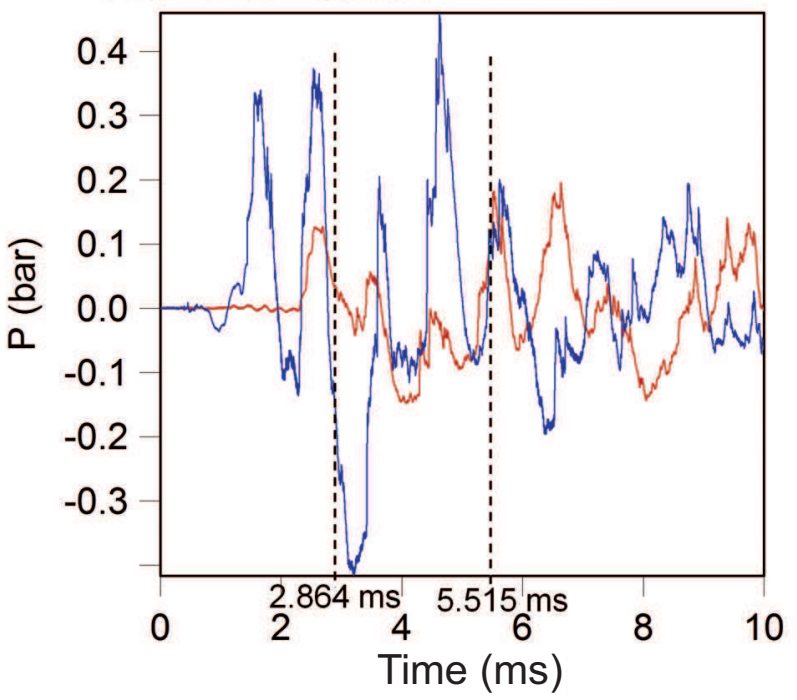

Fig. 32 Pressure histories recorded at gauges G1 and G14 in configuration 2.1 roofless for a $67 \mathrm{~mm}$ radius gaseous charge

Table 5 Differences between incident and maximal overpressures in configuration 2.1 and in free field at gauges G1 and G14

\begin{tabular}{|c|c|c|c|}
\hline \multirow{2}{*}{ Configuration } & $\begin{array}{c}\text { Charge } \\
\text { radius } \\
(\mathrm{mm})\end{array}$ & $\frac{\Delta P_{\max }}{\Delta P_{\text {free }}}(G 1)$ & $\frac{\Delta P_{\text {max }}}{\Delta P_{\text {free }}}(G 14)$ \\
\hline \multirow{3}{*}{2.1} & 30 & 1.807 & 1.594 \\
\cline { 2 - 4 } & 47 & 1.706 & 1.685 \\
\cline { 2 - 4 } & 67 & 1.619 & 2.405 \\
\hline
\end{tabular}

Table 6 Differences between scaled total positive impulse in configuration 2.1 and in free field at gauges G1 and G14

\begin{tabular}{|c|c|c|c|}
\hline \multirow{2}{*}{ Configuration } & $\begin{array}{c}\text { Charge } \\
\text { radius } \\
(\mathrm{mm})\end{array}$ & $\frac{I^{+}{ }_{\text {tot }}}{I^{+}{ }_{i}}(G 1)$ & $\frac{I^{+}{ }_{t o t}}{I^{+{ }_{i}}}(G 14)$ \\
\hline \multirow{3}{*}{2.1} & 30 & 119 & 137 \\
\cline { 2 - 4 } & 47 & 78 & 91 \\
\cline { 2 - 4 } & 67 & 48 & 63 \\
\hline
\end{tabular}

Unlike configuration 1, the increase in the maximal overpressure is more critical at gauge G14 (140\%) than at gauge G1 (61\%) in configuration 2.1 only for the largest charge. The reduction of the volume of cells 1 and 4 seems to have an impact on the protective effect associated with the longer distance from the charge by reinforcing it for small charge. Nevertheless, the increase in the total positive impulse is still more significant at gauge G14 (by a factor of 97 on average) than at gauge G1 (by a factor of 82 on average). 
A further reduction of the volume does not seem to change the phenomenology encountered in configurations 1 and 2.1. The pressure histories recorded at gauges G1 and G14 in confined environment show once more an increase in the maximal overpressure compared to the free field ( $76 \%$ for G1 and $115 \%$ for G14, Figure $33)$. The analysis of the tests conducted in this configuration without a roof (Figure 34) confirms that the maximal overpressure obtained at these two sensors is resulting from an interaction of the shock wave with the roof.

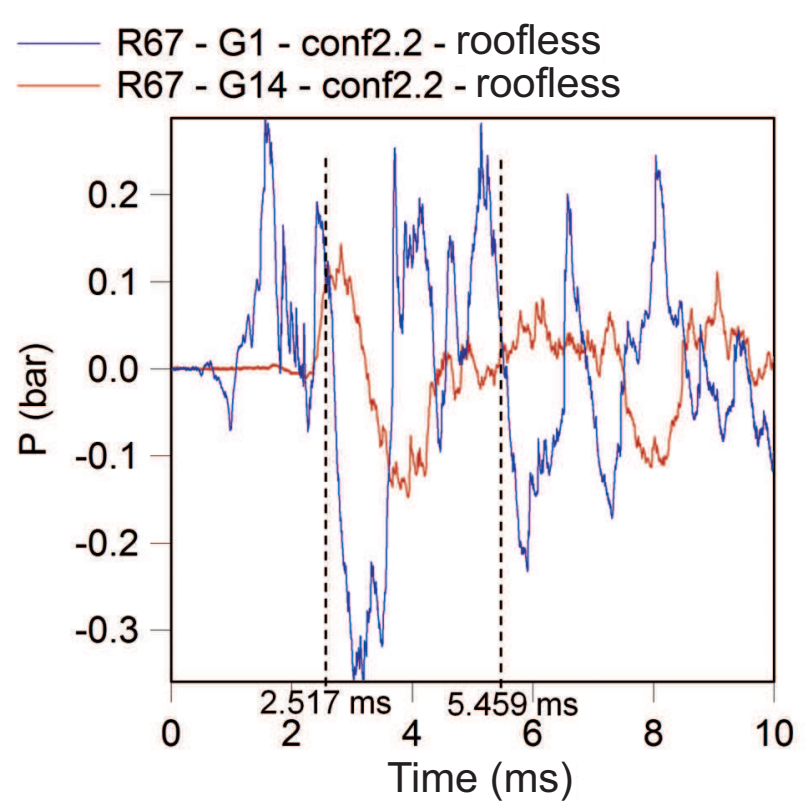

Fig. 33 Pressure histories recorded at gauges G1 and G14 in configuration 2.2 and in free field for a $67 \mathrm{~mm}$ radius gaseous charge

The numerical values of the differences observed for the maximal overpressure and total positive impulses between configuration 2.2 and free field are shown in tables 7 and 8 .

Table 7 Differences between incident and maximal overpressures in configuration 2.2 and in free field at gauges G1 and G14

\begin{tabular}{|c|c|c|c|}
\hline Configuration & $\begin{array}{c}\text { Charge } \\
\text { radius } \\
(\mathrm{mm})\end{array}$ & $\frac{\Delta P_{\max }}{\Delta P_{\text {free }}}(G 1)$ & $\frac{\Delta P_{\max }}{\Delta P_{\text {free }}}(G 14)$ \\
\hline \multirow{3}{*}{2.2} & 30 & 1.553 & 1.029 \\
\cline { 2 - 4 } & 47 & 1.389 & 1.476 \\
\cline { 2 - 4 } & 67 & 1.763 & 2.153 \\
\hline
\end{tabular}

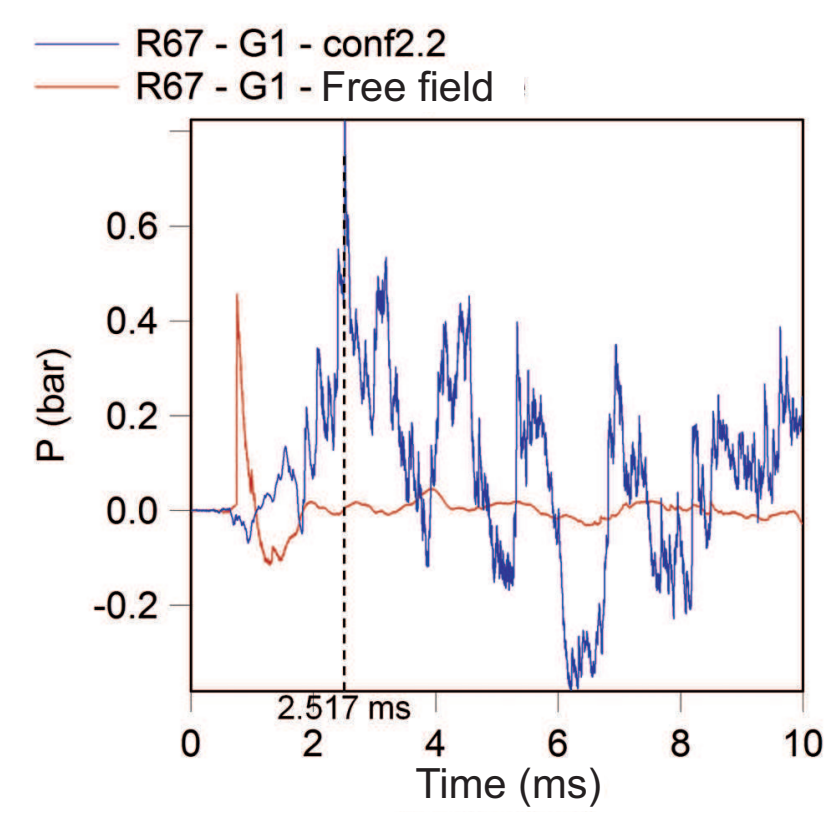

R67 - G14 - conf2.2

R67 - G14 - Free field

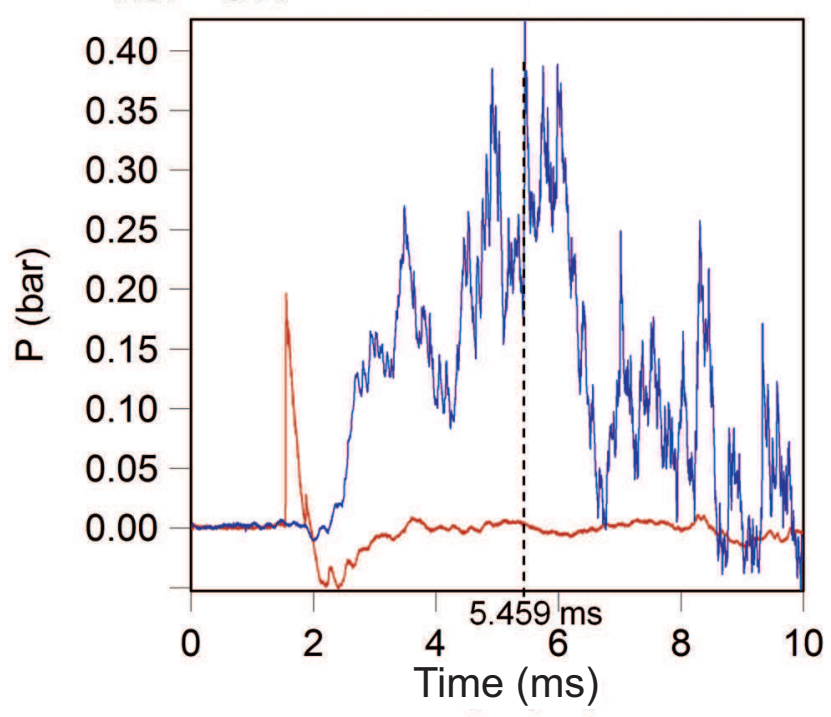

Fig. 34 Pressure histories recorded at gauges G1 and G14 in configuration 2.2 roofless for a $67 \mathrm{~mm}$ radius gaseous charge

This configuration presents an identical evolution to that of configuration 1 with a weaker increase in the maximal overpressure at gauge G14 (3\%) than at gauge G1 (55\%) for the $30 \mathrm{~mm}$ radius charge. This further reduction in volume compared to configuration 2.1 leads back to the behavior encountered in configuration 1 .

It is clear from this analysis that the confinement reduces the protective effect provided by the longer distance from the charge by reinforcing the maximal overpressure and the positive impulse. Nevertheless, an 
Table 8 Differences between scaled total positive impulse in configuration 2.2 and in free field at gauges G1 and G14

\begin{tabular}{|c|c|c|c|}
\hline Configuration & $\begin{array}{c}\text { Charge } \\
\text { radius } \\
(\mathrm{mm})\end{array}$ & $\frac{I^{+}{ }_{\text {tot }}}{I^{+}{ }_{i}}(G 1)$ & $\frac{I^{+}{ }_{\text {tot }}}{I^{+}{ }_{i}}(G 14)$ \\
\hline \multirow{3}{*}{2.2} & 30 & 119 & 156 \\
\cline { 2 - 4 } & 47 & 78 & 78 \\
\cline { 2 - 4 } & 67 & 48 & 61 \\
\hline
\end{tabular}

initial reduction of the volume of cells 1 and 4 from $0.028 \mathrm{~m}^{3}$ to $0.019 \mathrm{~m}^{3}$, that is to say moving from configuration 1.3 to configuration 2.1, seems to counter this phenomenon by requiring heavier charges to observe the loss of the protective effect. Yet, a second reduction of the volume of cells 1 and 4 from $0.019 \mathrm{~m}^{3}$ to $0.011 \mathrm{~m}^{3}$, observed when moving from configuration 2.1 to configuration 2.2 amplifies the loss of this protective effect.

The similarity of cells 1 and 4 has also been investigated through the analysis of all the pressure histories recorded in these cells, but this process is not presented in this article. This shows that the maximal overpressure as well as the total positive impulse have a more critical increase, compared to the free field, in the cell located the farthest from the charge. This may be linked to a channeling effect of the corridor.

\subsection{Phenomenological study of the corridor}

Gauges G4, G12 and G16 are gathered in the corridor. Gauge G8 is excluded from this area as it is in direct view of the charge and therefore not subjected to the same kind of wave as the other sensors placed in the corridor.

Pressure histories recorded at gauges G4, G12 and G16 in free field and in configuration 1.3 are plotted in figure 35. The incident overpressure is weaker than in free field for these three gauges ( $58 \%$ on average) while the associated arrival time is more important. This is due to the fact that these sensors are shielded from the charge by the structure of the model. The maximal overpressure is always linked to a reflected peak and is above the free field value (107\% on average). Gauges G4 and G12 have very similar pressure histories up to $1.5 \mathrm{~ms}$ (16 $\mu$ s maximal gap in arrival times and $4 \%$ in amplitudes). The similarity of the location of this two gauges relatively to the charge is the reason for this behavior.

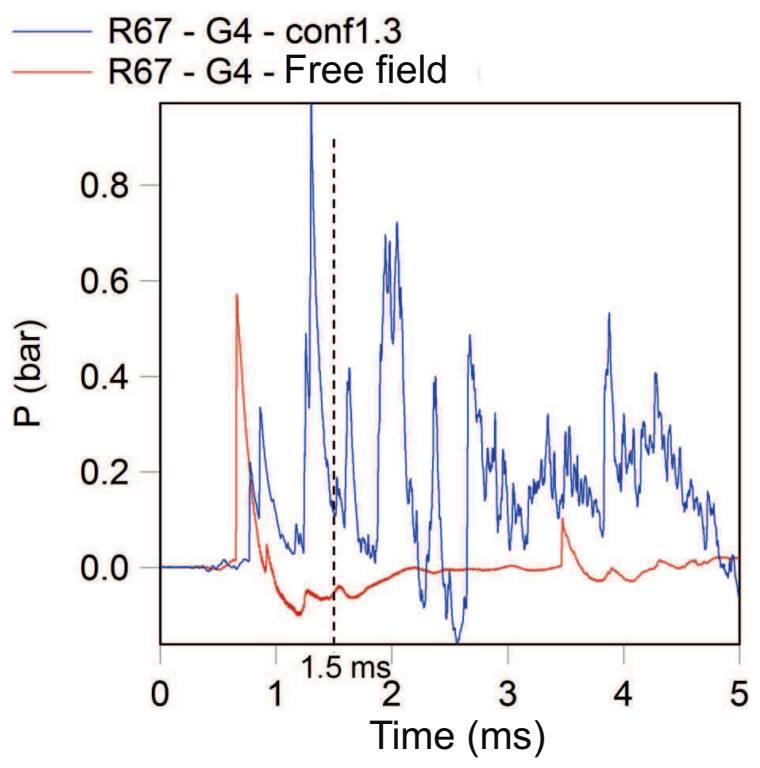

R67 - G12 - conf1.3

R67 - G12 - Free field

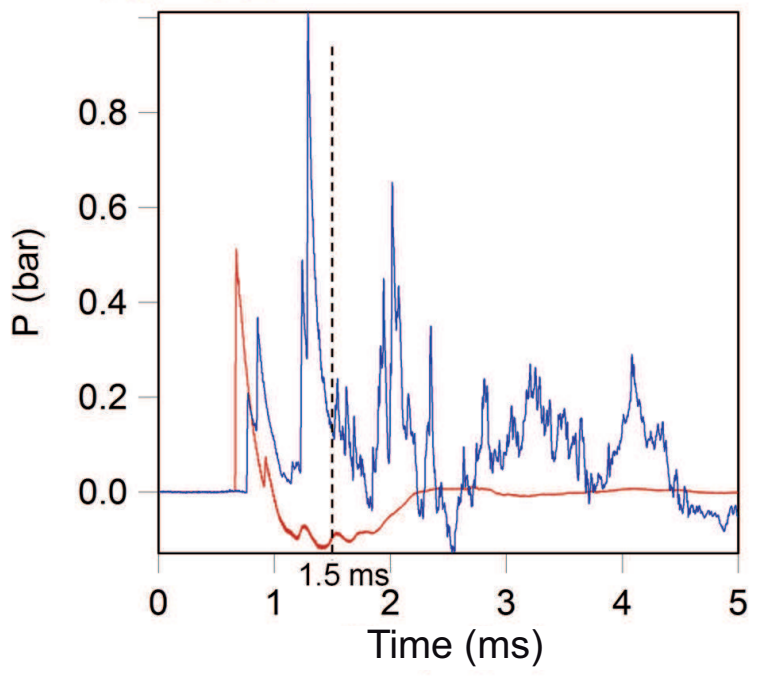

$$
\text { R67 - G16 - conf1.3 }
$$

R67 - G16 - Free field

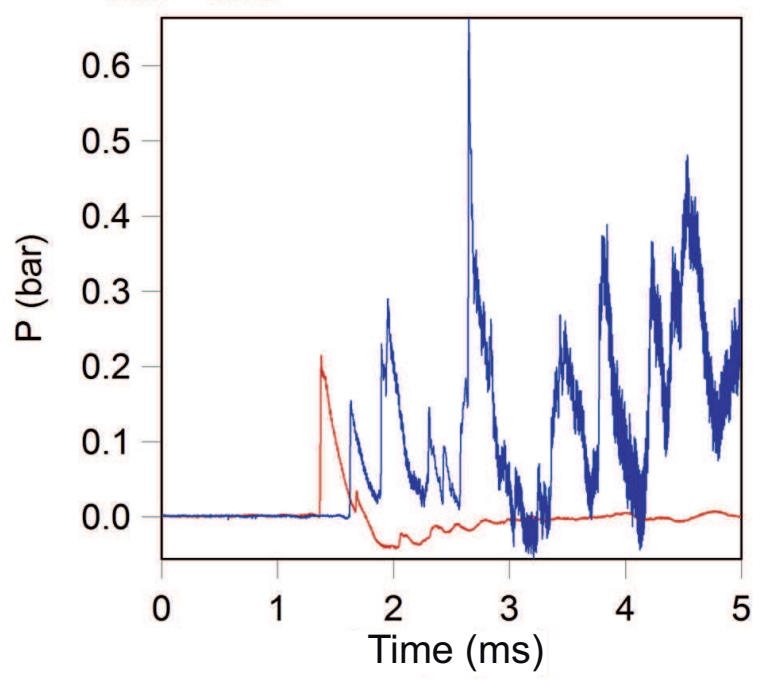

Fig. 35 Pressure histories recorded at gauges G4, G12 and G16 in configuration 1.3 for a $67 \mathrm{~mm}$ radius gaseous charge 


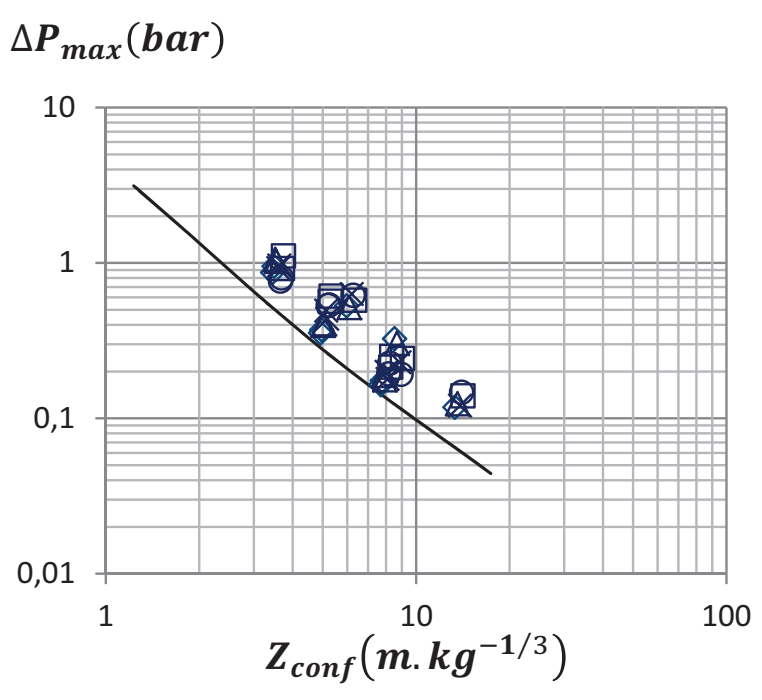

Fig. 36 Evolution of the maximal overpressure in the corridor in free field and in the model as a function of the scaled distance in the model (configurations $1.1(\diamond), 1.2(\triangle), 1.3$ $(\times), 1.4(\circ), 1.5(\square)$ and free field $(-))$

In roofless conditions, the maximal overpressure is reduced ( $24 \%$ on average) from the free field value. Consequently, the phenomena leading to the maximal overpressure come from interaction of the shock wave with the roof.

The influence of the width of the corridor can be studied through the analysis of the evolution of the maximal overpressure in configuration 1 . In this configuration, the width of the corridor ranges from $182 \mathrm{~mm}$ in configuration 1.1 to $59 \mathrm{~mm}$ in configuration 1.5. Yet, no simple evolution law as a function of the corridor width was found (Figure 36).

The pressure histories obtained at gauges G4, G12 and G16 in configurations 1.3 and 2.1 (Figure 37) are compared to evaluate the effect of the variation of the volume of the detonation cell on the propagation of the shock wave in the corridor.

The time difference observed on the pressure histories between configurations 1.3 and 2.1 is explained by the change in the sensors location.

The shape of the incident shock wave is identical in both configurations in the whole corridor. The increase in the detonation cell volume seems to have no noticeable effect on the incident shock wave.

The maximal overpressure has the same origin for gauges G4 and G16 since the maximum overpressure peaks show the same shape and have almost the same arrival time in both configurations. More precisely, these peaks appears for gauge G14 at $1.304 \mathrm{~ms}$ in configu-

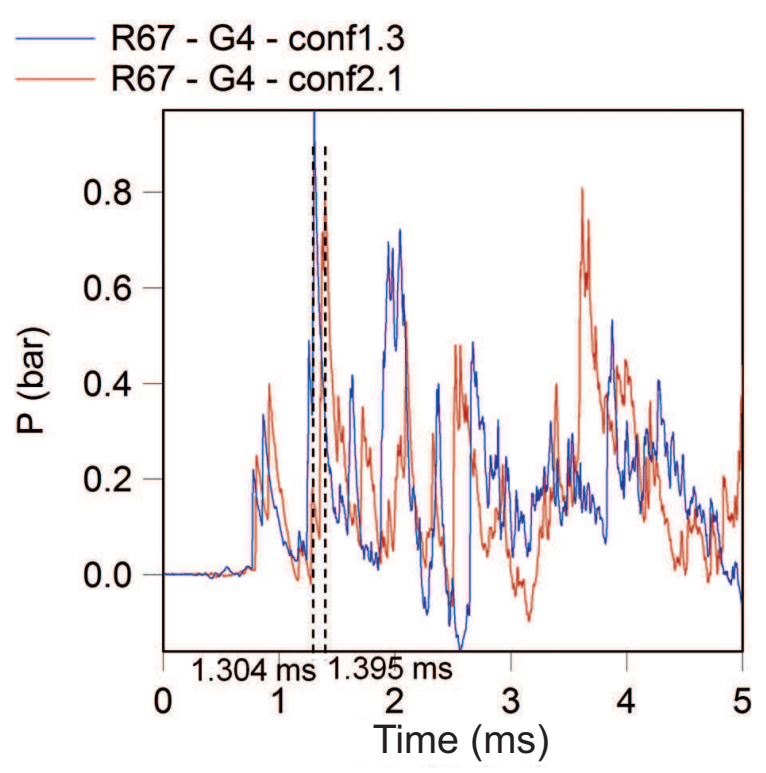

R67 - G12 - conf1.3

R67 - G12 - conf2.1

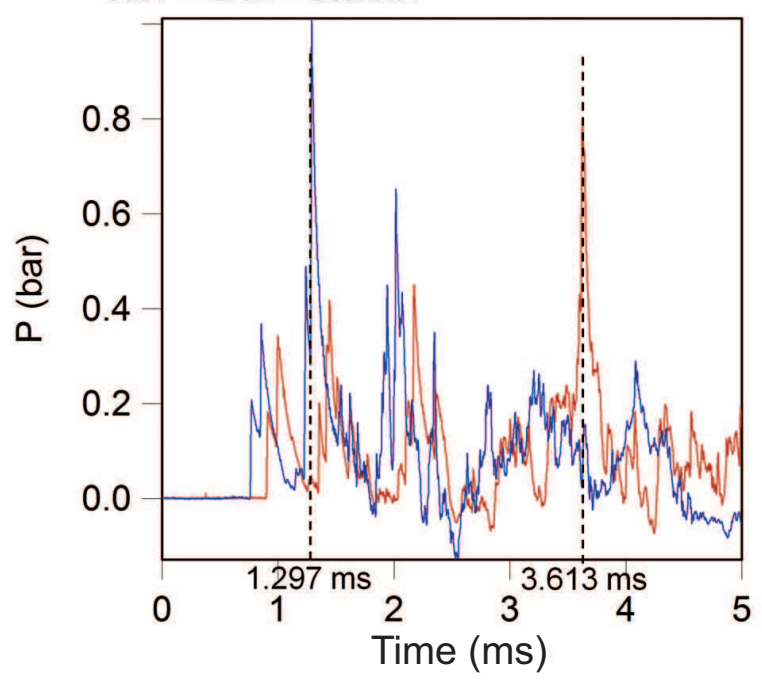

R67 - G16 - conf1.3

R67 - G16 - conf2.1

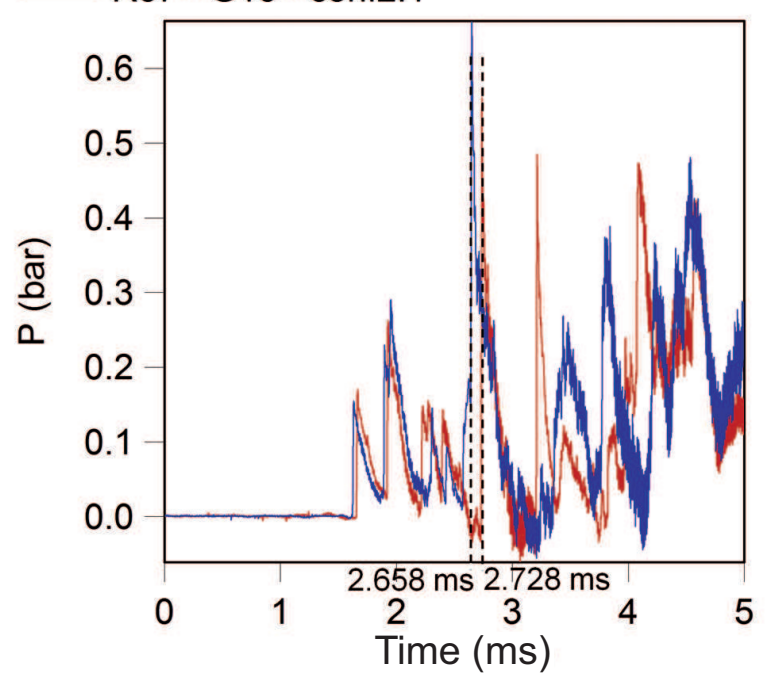

Fig. 37 Pressure histories recorded at gauges G4, G12 and G16 in configurations 1.3 and 2.1 for a $67 \mathrm{~mm}$ radius gaseous charge 
ration 1.3 and at $1.395 \mathrm{~ms}$ in configuration 2.1 and, for gauge G16, at $2.658 \mathrm{~ms}$ in configuration 1.3 and at $2.728 \mathrm{~ms}$ in configuration 2.1. On the other hand, the maximal overpressure recorded at gauge G12 in configuration 2.1 comes from a pressure peak arriving later (3.613 ms) than in configuration 1.3 (1.297 ms). However, the more important change in position between the two configurations for gauge G12 (84 mm) than for gauges G4 and G16 (42 mm) may be responsible for that.

The numerical values of the differences between the free field and configurations 1.3, 2.1 and 2.2 are gathered in table 9 . The increase in volume of the detonation cell seems to produce higher pressure level in the corridor.

Table 9 Differences between maximal overpressures in configuration 1.3, 2.1 and 2.2 and in free field at gauges G4, G12 and G16

\begin{tabular}{|c|c|c|c|c|}
\hline \multirow{3}{*}{ Conf } & $\begin{array}{c}\text { Charge } \\
\text { radius } \\
(\mathrm{mm})\end{array}$ & $\frac{\Delta P_{\max }}{\Delta P_{\text {free }}}(G 4)$ & $\frac{\Delta P_{\max }}{\Delta P_{\text {free }}}(G 12)$ & $\frac{\Delta P_{\max }}{\Delta P_{\text {free }}}(G 16)$ \\
\hline \multirow{3}{*}{1.3} & 30 & 1.237 & 1.352 & 1.729 \\
\cline { 2 - 5 } & 47 & 1.506 & 1.669 & 1.723 \\
\cline { 2 - 5 } & 67 & 1.841 & 1.735 & 2.77 \\
\hline \multirow{3}{*}{2.1} & 30 & 1.317 & 1.387 & 1.95 \\
\cline { 2 - 5 } & 47 & 1.742 & 1.494 & 2.13 \\
\cline { 2 - 5 } & 67 & 1.702 & 1.819 & 2.722 \\
\hline \multirow{3}{*}{2.2} & 30 & 1.294 & 1.279 & 2.223 \\
\cline { 2 - 5 } & 47 & 1.783 & 1.994 & 3.482 \\
\cline { 2 - 5 } & 67 & 2.17 & 1.533 & 2.599 \\
\hline
\end{tabular}

\section{Impact of the volume on the shock wave parameters throughout the model}

The analysis of the shock wave propagation throughout the model is much more complex than in cell 2 . This was already shown in the phenomenological analysis presented in section 4 . Sensors that are not in direct view of the charge may indeed present numerous reflections before reaching the maximal overpressure and it is almost impossible to find the origin of all of these peaks. Therefore, we chose to evaluate the impact of the volume on the shock wave parameters by looking at the evolution of these parameters with the volume of the cells.

\subsection{Arrival time}

Using the new set of scaled distances adapted to the multi-chamber system, the arrival time can be plotted as a function of $Z_{\text {conf }}$ in the whole model (Figure 38). The graph shows a very good fit between the scaled ar-

$$
\overline{t_{a}}\left(m s . k g^{-1 / 3}\right)
$$

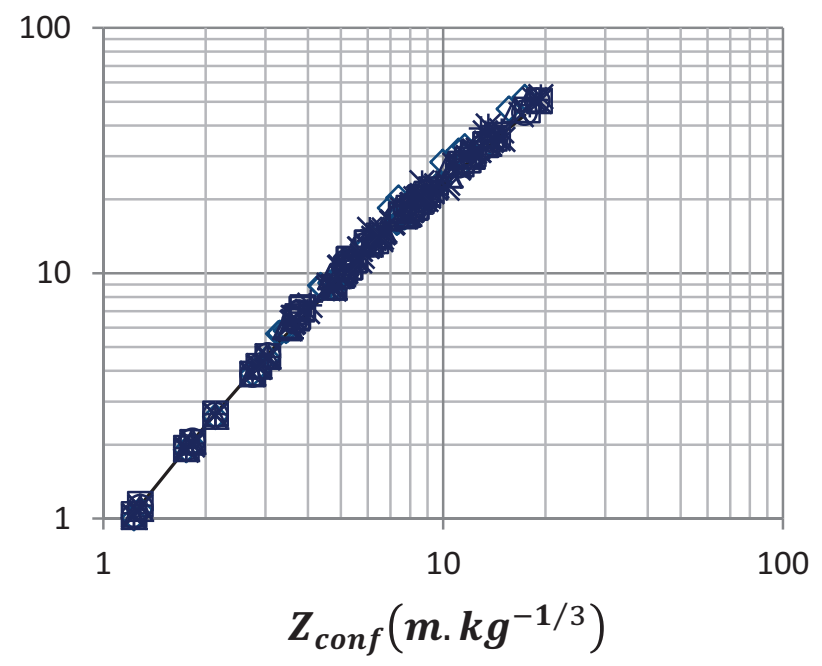

Fig. 38 Scaled arrival time versus scaled distance in the model and in free field

rival time measured in the model and the reference established in free field using the scaled distances adapted to a confined structure. The arrival time can therefore be predicted by equation (7) for all the volumes evaluated in our experiments.

$$
\begin{aligned}
\ln \left(\overline{t_{a}}\right)=- & 0.3216+1.8031 \ln \left(Z_{\text {conf }}\right)-0.0887 \ln \left(Z_{\text {conf } f}\right)^{2} \\
& -0.0368 \ln \left(Z_{\text {conf }}\right)^{3}+0.0081 \ln \left(Z_{\text {conf }}\right)^{4}
\end{aligned}
$$

with $1.229 \leq Z_{\text {conf }}\left(m . \mathrm{kg}^{-1 / 3}\right) \leq 19.373$

\subsection{Incident overpressure}

The approach based on the analysis of the evolution of the shock wave parameters does not resolve all issues. Indeed, the incident overpressure is very difficult to determine for most sensors that are not in direct view of the charge, because of the perturbations that modify the first moments of the pressure history. These perturbations may partly come from the waves that go through the internal walls of the model. Contrary to the hypothesis used in this work, the walls are not inelastic, unbreakable and perfectly reflecting. Part of the 


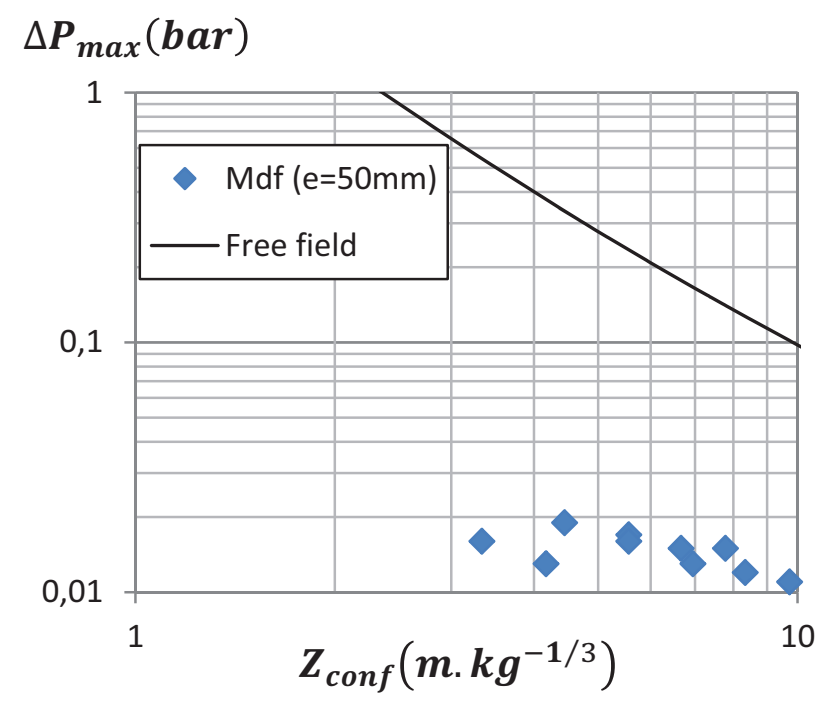

Fig. 39 Effect of a $50 \mathrm{~mm}$ thick medium density fiberboard (Mdf) wall on the maximal overpressure

wave passes therefore through the wall. A study was conducted at PRISME laboratory to evaluate the reflection and attenuation coefficient of several materials subjected to a gaseous detonation. Behind a $50 \mathrm{~mm}$ thick MDF wall, the maximal overpressure decreased by more than $90 \%$ (Figure 39).

Nevertheless, it can be said that the incident overpressure is always below or equal to the free field value that would be obtained for a sensor placed in free field at the same distance than in the model, that is to say for $Z=Z_{\text {conf }}$. This means also that the incident overpressure is always below or equal to the free field value, indicating the protective effect of the confinement on the incident shock wave (Figure 40).

\subsection{Maximal overpressure}

In every volume evaluated and for all of the pressure transducers, the maximal overpressure is always above or equal to the free field value that would be obtained for a sensors placed in free field at $Z=Z_{\text {conf }}$ (Figure 41). That means that the confinement produces higher pressure level than what could be observed in free field for a shock wave travelling the same distance.

The analysis of the maximal overpressure for each sensor revealed that it was necessary to gather the data by cells in order to find a global evolution for this parameter. We then chose to select only the maximal values of the maximal overpressure in each cell and to express the evolution of this new value as a function of the cubic root of the volume of the cell over the mass of the charge

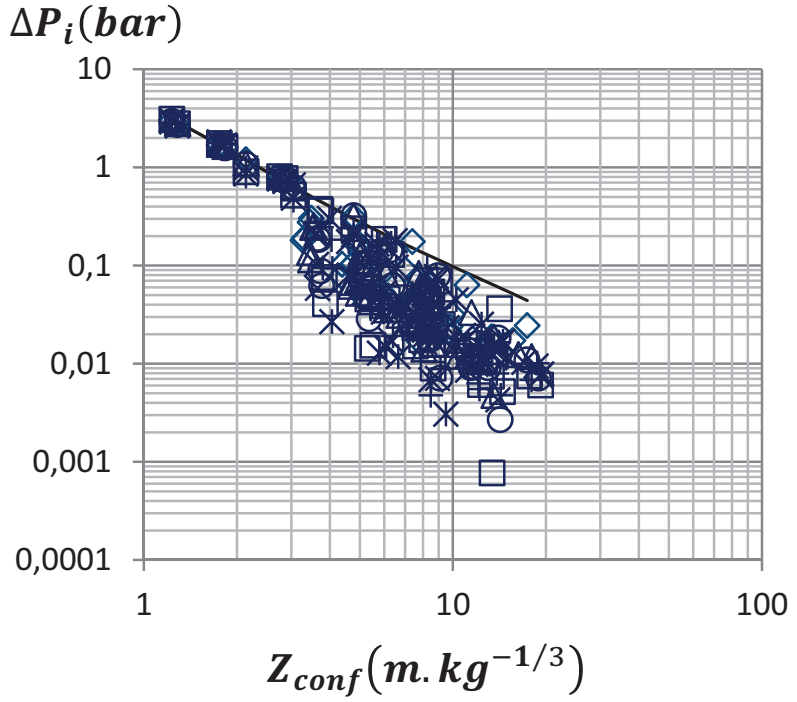

Fig. 40 Evolution of the incident overpressure in confined environment and in free field as a function of the scaled distance in the model (configurations $1.1(\diamond), 1.2(\triangle), 1.3(\times)$, $1.4(\circ), 1.5(\square), 2.1(+), 2.2(*)$ and free field $(-))$

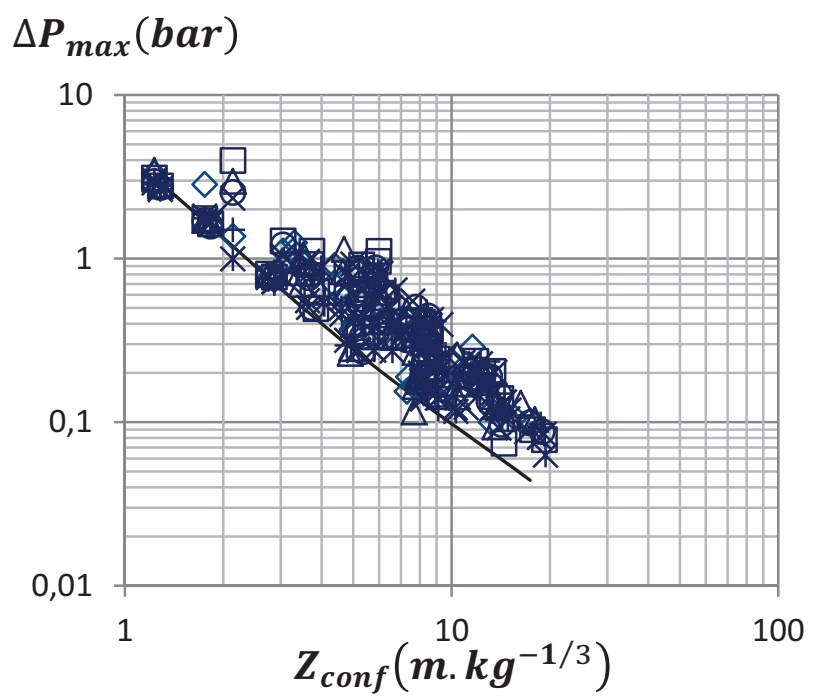

Fig. 41 Evolution of the maximal overpressure in free field and in the model as a function of the scaled distance in the model (configurations $1.1(\diamond), 1.2(\triangle), 1.3(\times), 1.4(\circ), 1.5$ $(\square), 2.1(+), 2.2(*)$ and free field $(-))$

$(V / M)^{1 / 3}$. In doing so, the distance from the charge no longer appeared in the equation. To take this into account, a new parameter was developed, $\left|y_{2-i}\right| / M^{1 / 3}$, where $y_{2-i}$ is the distance from the center of the charge to the center of cell i. Cell 2 being the detonation cell, the maximum overpressure in this cell is given by the detonation pressure which cannot compared to that of the other cells. Cell 2 is therefore excluded from this analysis.

The evolution of the maximal overpressure of cells 1,3 
and 4 as a function of $C=(V / M)^{1 / 3} \cdot\left|y_{2-i}\right| / M^{1 / 3}$ is plotted in figure 42 .

As can be seen on the graph, it seems that the evo-

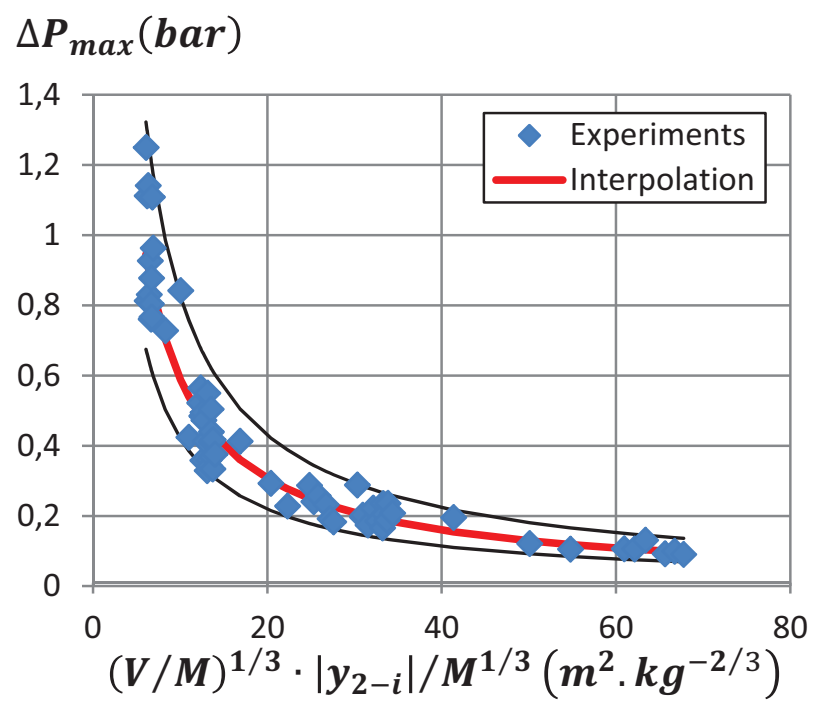

Fig. 42 Evolution of the maximal overpressure in the cells versus $(V / M)^{1 / 3} \cdot\left|y_{2-i}\right| / M^{1 / 3}$ in the model

lution of the maximal overpressure can be fitted by a power law. The value of the maximal overpressure in the cells is given by equation (8).

$\Delta P_{\max }=5.1898\left(\left(\frac{V}{M}\right)^{\frac{1}{3}} \cdot \frac{\left|y_{2-i}\right|}{M^{\frac{1}{3}}}\right)^{-0.944}$

with $6.079 \leq C\left(\mathrm{~m}^{2} \cdot \mathrm{kg}^{-2 / 3}\right) \leq 67.555$

A very good fit is observed with the experimental data with a regression coefficient $R^{2}=0.946$. A $90 \%$ confidence interval was plot in black on the graph (Figure 42).

The maximal overpressure can thus be calculated inside a cell with equation (8). We then looked at the corridor so that the maximal overpressure could be predicted in the whole model. To do so, we took the shape of non dimensional parameters used in tunnel [16] and adapted it to our configuration. Two normative parameters $\mathrm{A}$ and $\mathrm{B}$ were then defined to alter the value of the maximal overpressure and the scaled distance. These parameters are a function of the mass of the charge $\mathrm{M}$, the volume of the cell facing the sensor $\mathrm{V}$, and the reference volume $\mathrm{V}_{0}$ calculated for the detonation cell in configuration 1.3 .

$$
\begin{aligned}
& B=\frac{V}{V_{0}} \\
& A=\frac{M}{B}^{1 / 3}
\end{aligned}
$$

Figure 43 presents the evolution of the maximal overpressure in the corridor over A versus the scaled distance in the model over B.

It seems that the evolution of the maximal overpres-

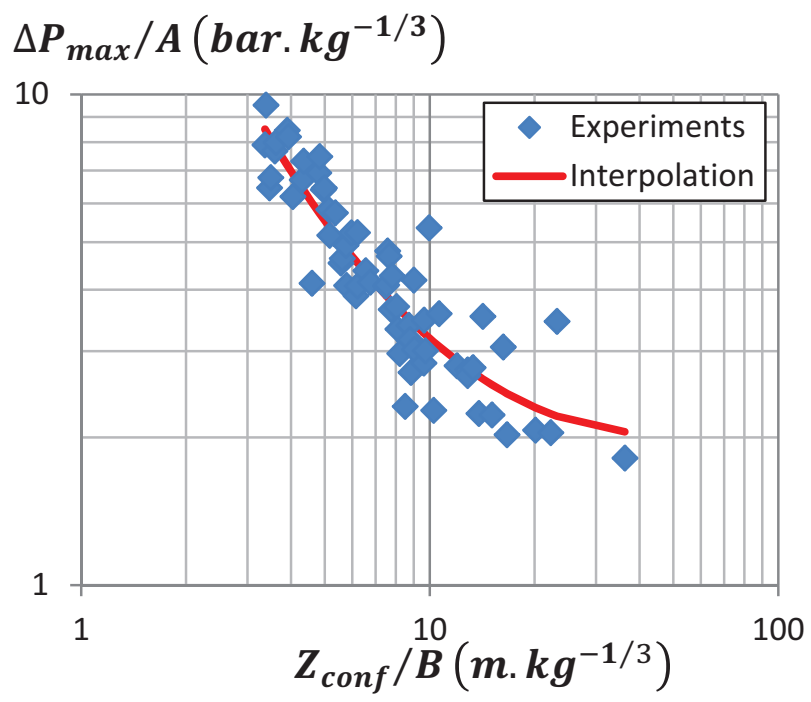

Fig. 43 Evolution of the maximal overpressure over A in the corridor as a function of the scaled distance in the model over $\mathrm{B}$

sure in the corridor can be fitted by a logarithmic law. The value of the maximal overpressure in the corridor is given by equation (11).

$$
\begin{aligned}
& \ln \left(\frac{\Delta P_{\max }}{A}\right)=4.026-1.9347 \ln \left(\frac{Z_{\text {conf }}}{B}\right) \\
& \quad+0.3298 \ln \left(\frac{Z_{\text {conf }}}{B}\right)^{2}-0.0132 \ln \left(\frac{Z_{\text {conf }}}{B}\right)^{3}
\end{aligned}
$$

with $3.367 \leq Z_{\text {conf }}\left(\mathrm{m} \cdot \mathrm{kg}^{-1 / 3}\right) \leq 36.312$

A comparison of the maximal overpressure calculated using equations (8) and (11) with numerical simulation was carried out to ascertain the validity of these laws. More precisely, it allowed us to verify that the positioning of the pressure transducers in the cells and the corridor was suitable to catch the maximal overpressure in these areas. These simulations were run using OURANOS CFD code.

A graphical presentation of the maximal overpressure obtained with numerical simulation for the detonation of a $67 \mathrm{~mm}$ radius charge in configuration 1.3 is given in figure 44 . The same results calculated with the prediction laws are gathered in table 10. For an easier comparison the maximal overpressure in the corridor has been evaluated for the areas facing the cells. It appears that the overpressure calculated with the laws are close 

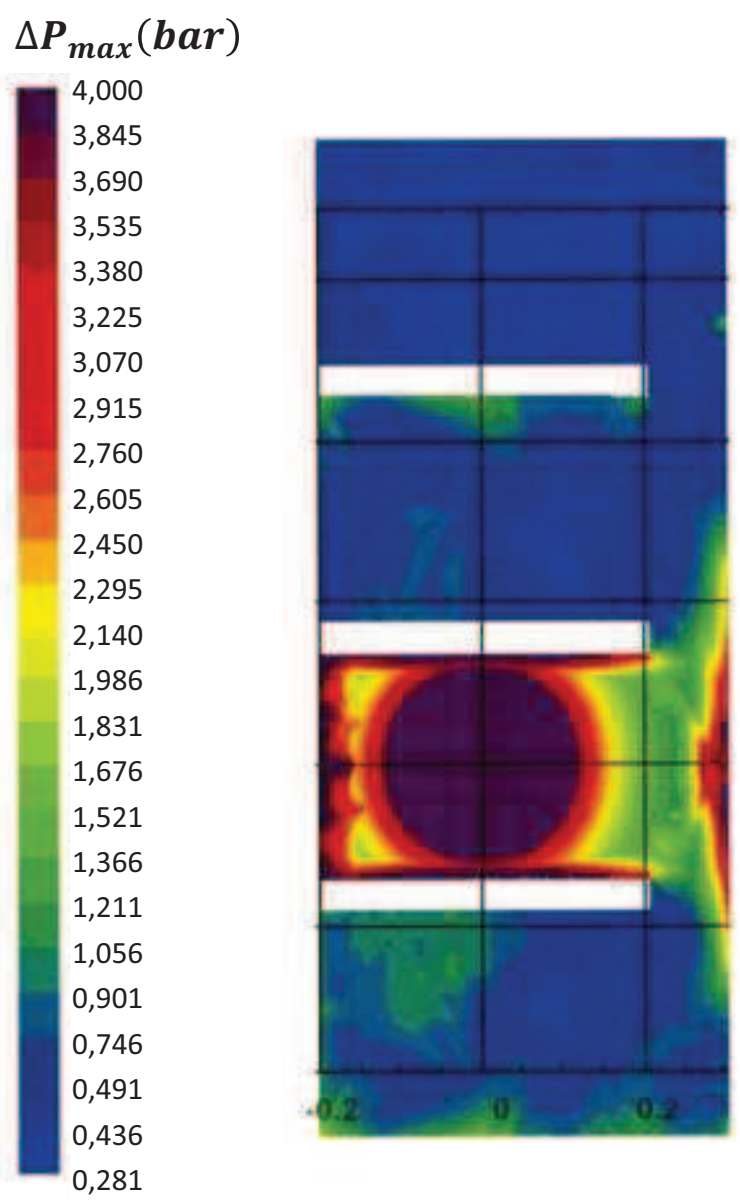

Fig. 44 Maximal overpressure obtained with numerical simulation for the detonation of a $67 \mathrm{~mm}$ radius gaseous charge in configuration 1.3

to the simulation results with a maximum gap of $20 \%$. The location of the sensors seems therefore to be adequate to measure the maximal overpressure in the cells as well as in the corridor. This was also verified for the other configurations and charges.

Table 10 Values of the maximal overpressure calculated with laws (8) and (11) in configuration 1.3 for a $67 \mathrm{~mm}$ radius gaseous charge

\begin{tabular}{|c|c|c|c|}
\hline Cells & $\Delta$ Pmax (bar) & Corridor & $\Delta$ Pmax (bar) \\
\hline Cell 1 & 0.88 & Area C1 & 1.968 \\
\hline Cell 3 & 0.867 & Area C3 & 1.93 \\
\hline Cell 4 & 0.456 & Area C4 & 0.662 \\
\hline
\end{tabular}

Regarding the arrival time associated with the maximal overpressure, it seems to be highly variable and shows no simple evolution pattern. Moreover, the peak leading to the maximal overpressure can occur after several reflected peaks ranging from 1 to 127 .

\subsection{Total positive impulse}

As can be expected due to the multiple reflections, the total positive impulse, integrated over the full signal period $(150 \mathrm{~ms})$, is much stronger than the positive impulse in free field (Figure 45).

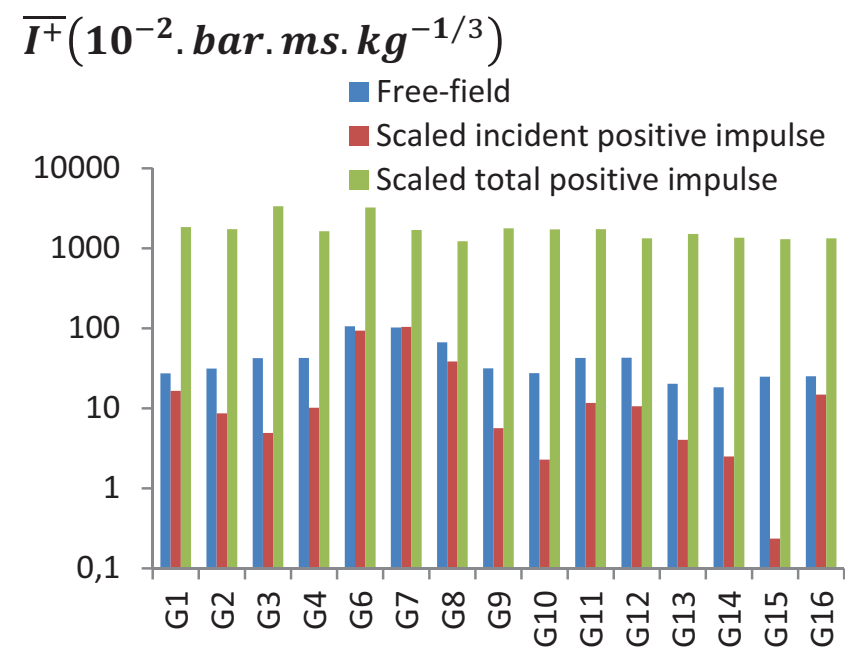

Fig. 45 Scaled incident positive impulse in free field compared with scaled incident and total positive impulses in the model for a $67 \mathrm{~mm}$ radius gaseous charge in configuration 1.3

The total positive impulse does not seem to present a simple evolution pattern with the scaled distance or the volume of the cells. Figure 45 has a misleading shape as the use of the logarithmic scale lead to believe that an average value can be found for the total positive impulse. Actually, there can be a factor 7 between the extreme values of this parameter.

Therefore, the approach that was presented previously for the maximal overpressure in the corridor has been applied to the total positive impulse. The evolution of the total positive impulse can then be given by equation (12). It gives variable results as it seems to fit only the pressure transducers located in cell 3 as illustrated by figure 46 .

$$
\frac{\overline{I^{+}}}{A}=f\left(\frac{Z_{\text {conf }}}{B}\right)
$$




$$
\overline{I^{+}} / \boldsymbol{A}\left(\text { bar. } \boldsymbol{m s} . \boldsymbol{k g}^{-2 / 3}\right)
$$
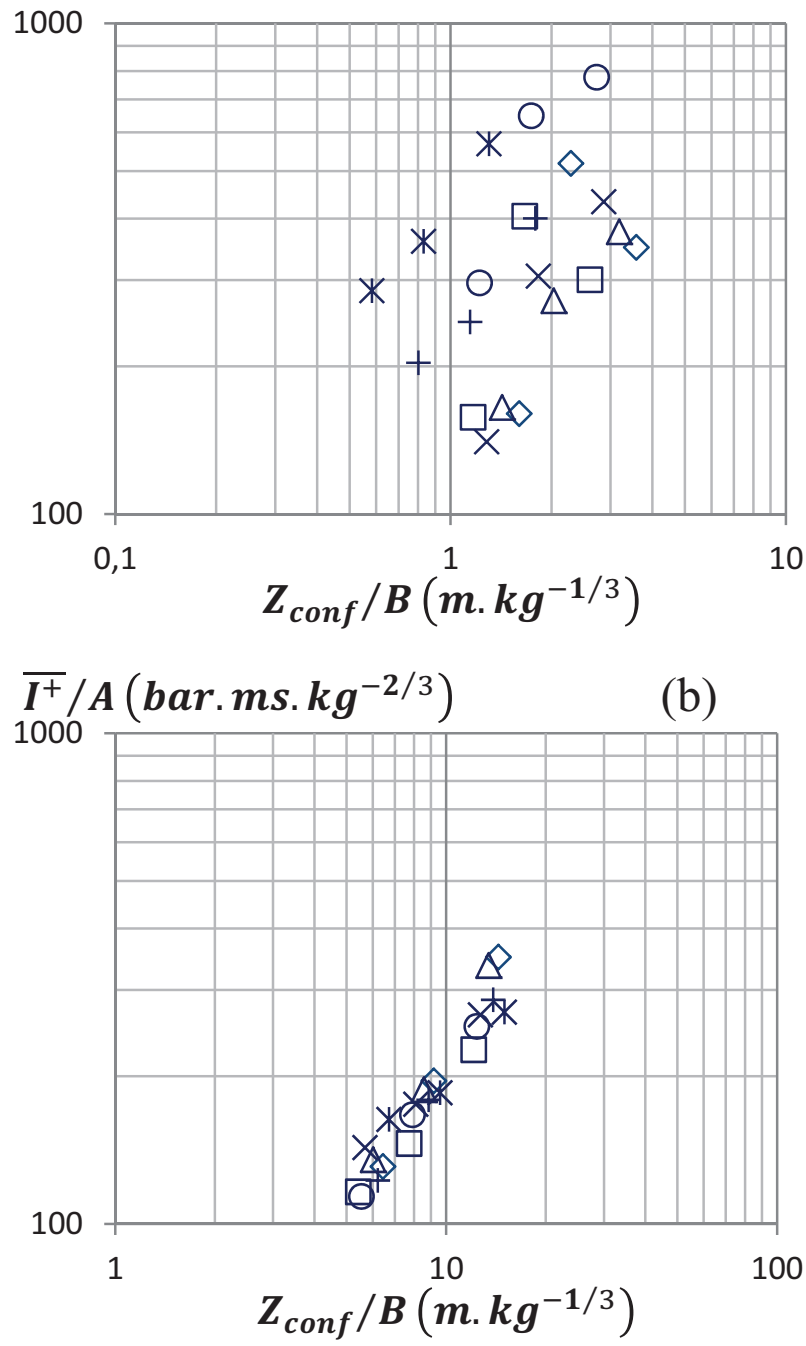

Fig. 46 Evolution of the scaled total positive impulse over $\mathrm{A}$ as a function of the scaled distance in the model over B at gauge G7 (a) and gauge G10 (b)(configurations $1.1(\diamond), 1.2$ $(\triangle), 1.3(\times), 1.4(\circ), 1.5(\square), 2.1(+), 2.2(*)$ and free field $(-))$

\section{Conclusions}

This study focuses on the behavior of a shock wave within a confined multi-chamber system and on the impact of different parameters such as the size of the rooms and the width of the corridor on the pressure history inside the building. The two configurations presented in this article are designed to evaluate the effect of the volume of the rooms on the shock wave propagation. Five different lengths are tested in the first configuration and three widths in the second.

Several preliminary small-scale experiments have been carried out using an adjustable model of a four-roomed single-story building, representative of a pyrotechnic workshop and a hemispherical charge of a propaneoxygen stoichiometric mixture. The shock wave propagation is analyzed through the evolution of some of the shock parameters (maximum overpressure and positive impulse).

The results show that the pressure histories within the model are very complex. Yet, we were able to determine the origin of some reflected peaks for the gauges in direct view of the charge.

The complexity of the pressure histories led us to a more global approach. In doing so, a very good fit with the free field reference curve was obtained for the arrival time. Moreover, by selecting only the maximum of the maximal overpressure in each cell, an evolution law for the maximal overpressure has been found for the cells in all of the evaluated volumes. Another law has been established for the maximal overpressure in the corridor. The pressure can therefore be estimated in the whole model as a function of the volume of the cell, except in the area where gauge G8 is positioned. This pressure transducers, being in direct view of the charge, presents indeed a different behavior from the other sensors of the corridor.

The validity and precision of the law established for the maximal overpressure of the cells is based on the fact that the gauges measure the actual maximum overpressure in each cell. This was ascertain with numerical simulations. A 20\% maximal gap was found between the prediction laws and numerical simulation, proving that the position of the sensors is adequate.

As could be expected, the most dangerous cell, regarding the maximal overpressure, is the one where the detonation is initiated. Yet, it has to be noticed that, due to the various propagation phenomena of the shock wave within the multi-chamber model, significant pressure levels may still be encountered in other cells of the model, even at the most distant parts. So that it appears that extended areas within the model may be associated to overpressures which could be high enough to be dangerous to human health $\left(\Delta P_{\max }>50 \mathrm{mbar}\right)$, or even lethal $\left(\Delta P_{\max }>430 \mathrm{mbar}\right)$.

Acknowledgements This work was funded by DGA Land Systems under contract $n^{\circ} 10-02-0288$ A.

\section{References}

1. U.S. Department of the Army, Fundamentals of protective design for conventional weapons, Technical Manual 5-1300 (1986)

2. U.S. Department of the Army, Structures to resist the effects of accidental explosions, Technical Manual 5-1300 (1990) 
3. G.F. Kinney, Explosive Shocks in Air, MacMillan, London (1962)

4. G. Schelinski-Glück, Blast Propagation in Tunnels behind Chambers from Cylindrical H.E-Charges Detonation in the Tunnel Entrance, $13^{\text {th }}$ Int. Symp. on Military Aspects of Blast and Simulation, The Hague, The Netherlands (1993)

5. R.C. Ripley, B. Von Rosen, D.V. Ritzel, D.R. Whitehouse, Small-scale modeling of explosive blasts in urban scenarios, $21^{\text {st }}$ Int. Symp. on Ballistics, Adelaide, Australia (2004)

6. H. Reichenbach, P. Neuwald, Fluid Dynamics of Explosion in Multi-chamber Systems Phenomenology Test Program, Defense Threat Reduction Agency Technical Report $\mathrm{n}^{\circ}$ ADA 384351 (2000)

7. H. Reichenbach, P. Neuwald, A.L. Kuhl, Role of Precision Laboratory Experiments in the Understanding of LargeScale Blast Phenomena, Julius J. Meszaros Lecture, $17^{\text {th }}$ Int. Symp. on Military Aspect of Blast and Shock, Las Vegas, Nevada, USA (2002)

8. P. E. Sauvan, I. Sochet, S. Trélat, Analysis of reflected blast wave pressure profiles in a confined room, Shock Waves, 22, 253-264 (2012)

9. B. Julien, I. Sochet, T. Vaillant, Explosion in a multichamber: Experimental investigation, $22^{\text {nd }}$ Int. Symp. on Military Aspect of Blast and Shock, Bourges, France (2012)

10. S. Trélat, I. Sochet, B. Autrusson, O. Loiseau, K. Cheval, Strong explosion near a parallelepipedic structure, Shock Waves, 16, 349-357 (2007)

11. R. Mainiero, M. Sapko, Blast and Fire Propagation in Underground Facilities, Defense Nuclear Agency technical report, contract $\mathrm{n}^{\circ}$ DNA JACRO-93-862, DNA-TR-93-159 (1996)

12. N. Sinha, B.G. Crawford, J.D. Ott, High fidelity simulations of complex multi-room target defeat using energetic explosives, $21^{\text {st }}$ Int. Symp. on Military Aspect of Blast and Shock, Jerusalem, Israel (2010)

13. S. M. Kogarko, V. V. Adushkin, A. G. Lyamin, Investigation of spherical detonation of gas mixtures, Fizika Goreniya i Vzryva, 1, 22-34 (1965)

14. T.A. Rose, P.D. Smith, Oblique Clearing : Blasts Loads on Buildings at Non-Zero Angles of Incidence, $18^{\text {th }}$ Int. Symp. on Military Aspect of Blast and Shock, Bad Reichenhall, Germany (2004)

15. Y. Gitterman, Air-blast analysis and secondary shock features for large-scale surface chemical explosions at Sayarim Military Range, Israel, $22^{\text {nd }}$ Int. Symp. on Military Aspect of Blast and Shock, Bourges, France (2012)

16. C.E. Joachim, C.V. Lunderman, Parameter study of underground ammunition storage magazines: result of explosion tests in small-scale models, Technical Report $n^{\circ}$ ADA 500131 (1994) 